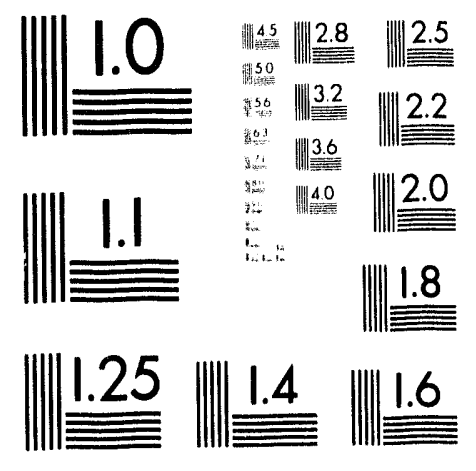



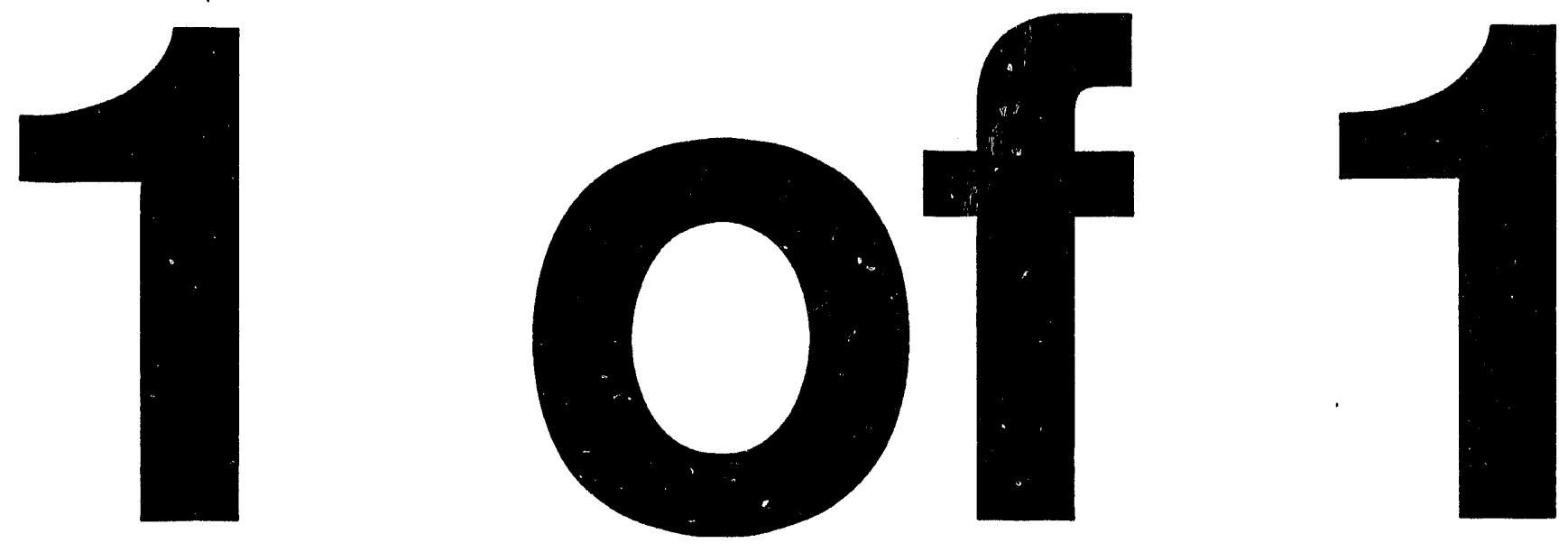


\title{
THEORY AND SIMULATION OF PLASMA SHEATH WAVES
}

\author{
by
}

\author{
X. Q. Xu, G. DiPeso, V. Vahedi, and C. K. Birdsall
}

Memorandum No. UCB/ERL M92/148

15 December 1992

DISCLAIMER

This report was prepared as an account of work sponsored by an agency of the United States Government. Neither the United States Government nor any agency thereof, nor any of their employees, makes any warranty, express or implied, or assumes any legal liability or responsibility for the accuracy, completeness, or usefulness of any information, apparatus, product, or process disclosed, or represents that its use would not infringe privately owned rights. Reference herein to any specific commercial product, process, or service by trade name, trademark, manufacturer, or otherwise does not necessarily constitute or imply its endorsement, recommendation, or favoring by the United States Government or any agency thereof. The views and opinions of authors expressed herein do not necessarily state or reflect those of the United States Government or any agency thereof.

\section{ELECTRONICS RESEARCH LABORATORY}

\author{
College of Engineering \\ University of California, Berkeley \\ 94720
}




\title{
Theory and Simulation of Plasma Sheath Waves
}

\author{
X. Q. Xu, G. DiPeso, V. Vahedi, C. K. Birdsall \\ Electronics Research Laboratory \\ University of California, Berkeley, California 94720
}

\begin{abstract}
Abatract
Sheath waves have been investigated analytically and with particle simulation for an unmagnetized two dimensional plasma slab with periodic boundary conditions in $y$ and conducting walls at $x=0, L_{x}$. Analytically treating the sheath as a vacuum layer, the sheath wave bears a resemblance to plasma vacuum surface waves. The simulations are in agreement with the theory for both bulk Bohm Gross waves and edge sheath waves, with some unanswered questions. Some waves that were expected did not show up, at least, where we thought they should be. Hence, improvements were made in the initialization (a better quiet start), in the diagnostics (especially the spectra in frequency), and in the excitation (ability to pulse). It has become cleas that this problem, seeking both sheath (or surface) and body waves in a bounded system, needs far more attention, in analysis (non-uniform density included) and in simulation, especially in diagnostics. Hence, this report is to be treated as a start on the problem. The problem is not dropped, as the understanding of such waves (in $2 \mathrm{~d}$ and $3 \mathrm{~d}$ ) is very important, for both basic sheath understanding and for applications, such as plasma control via excitation of sheath or pre-sheath waves.
\end{abstract}

\section{Introduction}

It is well known that there is a great variety of waves in a plasma that is well neutralized $\left(n_{i} \simeq n_{e}\right)$ and does not have sharp gradients in field or density quantities. Waves also exist at the plasma edge or sheath where there is large charge imbalance $\left(n_{i} \neq n_{e}\right)$ and where the gradient scale lengths are on the order of the electron Debye length in the unmagnetized case, or on the order of the ion gyroradius in the magnetized case. These waves have received less attention in the literature. This paper is a report on electrostatic waves propagating along the unmagnetized plasma edge or sheath. Both analytic theory and computer simulation are used to study the sheath waves. The computer simulation may be viewed as an computer experiment as the simulation model is constructed from 
first principal physics with a minimum of approximations or assumptions.

Before we start on the two dimensional theory and simulations, let us review the results of one dimensional simulations [1]. The one dimensional simulations are bounded by perfectly conducting walls which are connected by an external RLC circuit with optional voltage and current sources (see Fig. 1a). The simplest boundary conditions for which sheath formation is observed is the short circuit where the reference potential or voltage on both walls is set to zero. The device is initially filled with warm electrons at a density $n_{e 0}$. The electrons have a full Maxwellian velocity distribution at a temperature $T_{e}$. The ions are treated as an immobile background with a density $n_{i 0}=n_{\mathrm{e}}$ so that the system is initially neutral. The device length is about $50 \lambda_{D e}$.

During the first few time steps of the simulation, some of the faster electrons flow to both walls (see Fig. $1 \mathrm{~b}$ and $1 \mathrm{c}$ ) and are lost, leaving a net positive charge in the plasma near the walls. The potential in the center rises to $e \phi_{\text {middle }} \simeq$ few $T_{e}$ (see Fig. 1d). The remaining electrons are trapped by the repelling fields of the sheaths at the absorbing walls (see Fig. 1e). The sheath, with enforced uniform ion density, is sometimes called a "matrix sheath". Oscillations in $\phi_{\text {middle }}$ are observed at a frequency close to $\omega_{\text {pe }}$ (see Fig. If and 1g). Oscillations are observed in the external current at the series resonant frequency[2] associated with the $k_{y}=0$ (cutoff) asymmetric mode (see Fig. $1 \mathrm{~h}$ and 1i). We also found that some slow particles are trapped in localized phase space vortices, possibly due to standing Bohm-Gross waves.

Repeating the same initial conditions in a model with mobile ions (protons) give much the same results except that both species gradually went to the walls.

The two-dimensional computer experiment is expected to behave much the same way. What we have done is to find waves propagating near and along the wall (not allowed in the 1d model), separate from bulk plasma waves. Plasma surface waves in general may be excited when the plasma is in contact with another medium, e.g., a vacuum or a conducting wall. Classical examples of these waves inciude the Gould-Trivelpiece (G-T) modes in plasma filled waveguides [3]. A sheat'd wave is a surface wave specific to the case of a plasma bounded by a conducting wall. This wave is somewhat similar to the surface wave occuring at a vacuum plasma interface $[4,5]$.

Recent interest involving surface waves has been motivated by anomalous impurity concentrations and edge heating observed in tokamak ion Bernstein wave experiments [6]. A possible explanation for the observed anomaly has been given as an additional class of waves near the antenna, sheath-plasma waves(SPW), which propagate on the high-voltage of sheath driven by the antenna [7]. The L-H mode transition observed in tokamaks, controllable by biases on divertor plates, implies edge plasma control of the bulk plasma ( $n$ and $T_{e}$ ) profiles[9]. Sheath waves also may have effects on sheath formation in plasma processing chambers and thus merit further study. And, of course, sheath and 


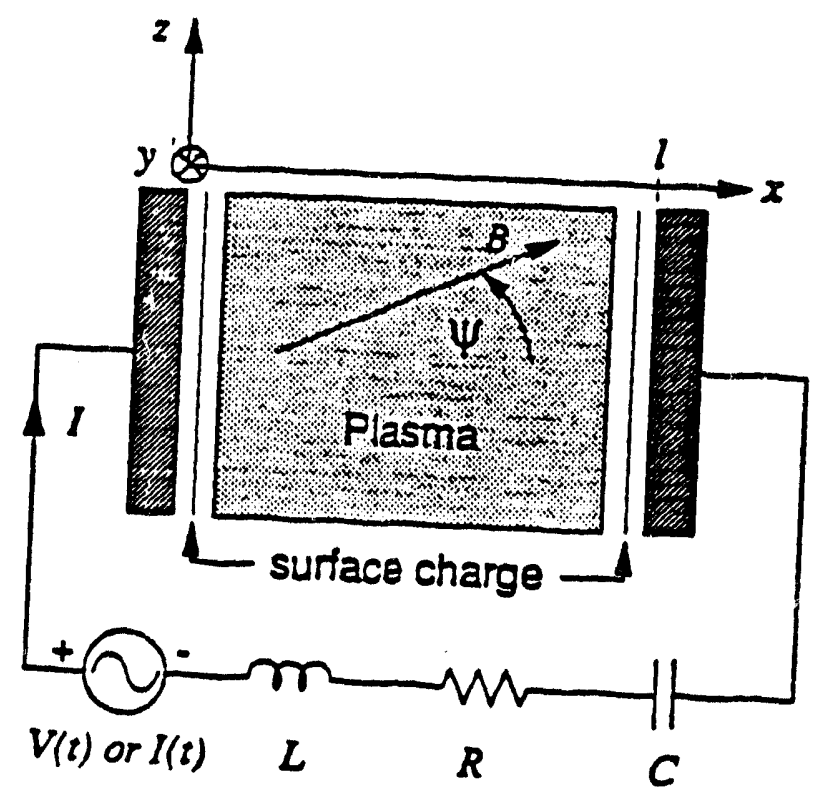

Figure 1: Id bounded simulation model and resule. Fig la. Model; the external circuit used was a short circuit, and $B=0 . L=0.01 \mathrm{~m}$, and $n_{0}=1 \times 10^{1 \mathrm{~s} / \mathrm{m}^{3}}$; the ions are immobile. 

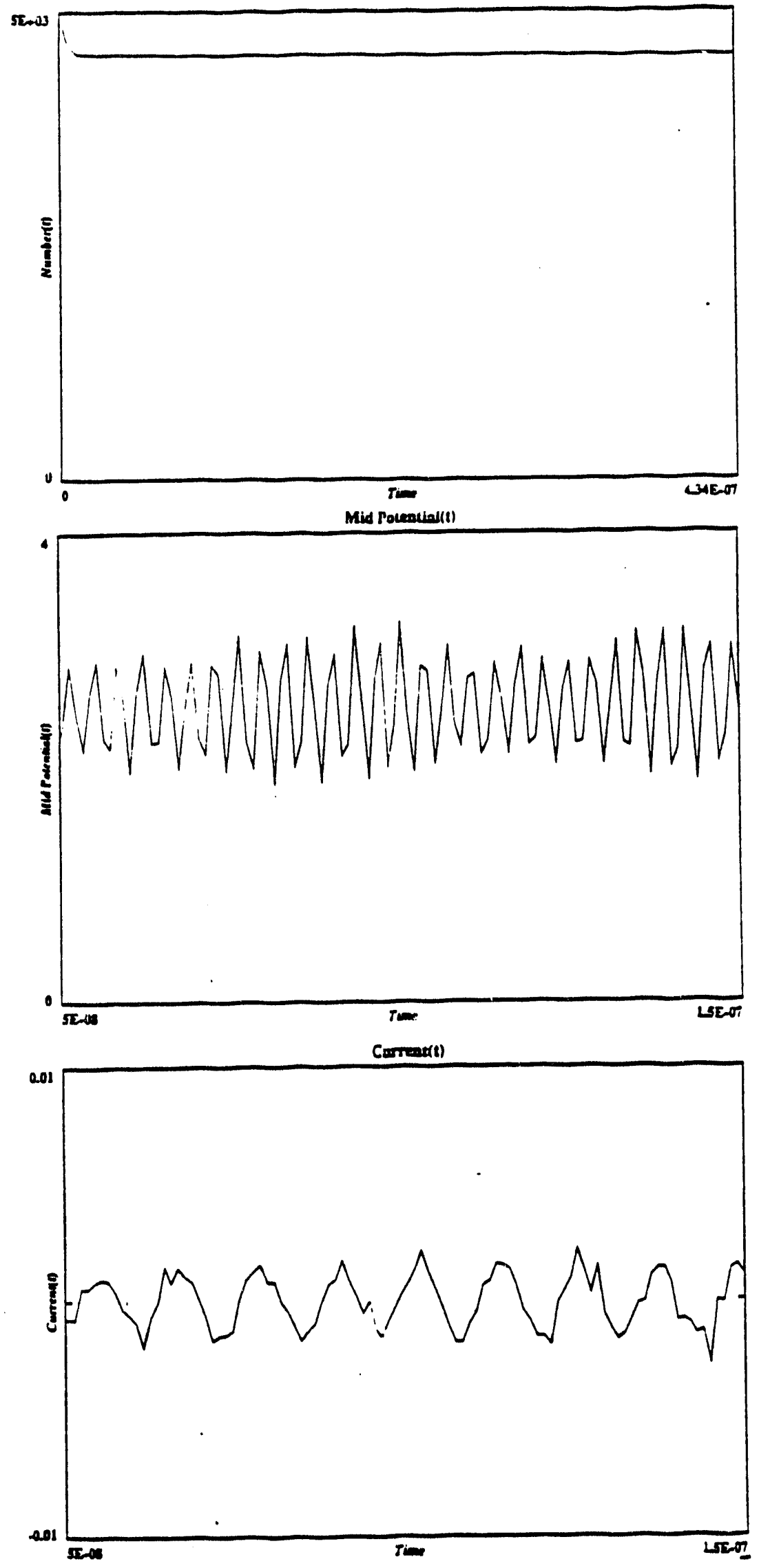

Figure 1: 1d bounded simulation model and resule Fig lb. Number of simulation particles (electrons) vs. ime; note that the electrons are trapped after the sheach is formed. Fig If. Time history of midpotential. Fig it. Time history of the external current 

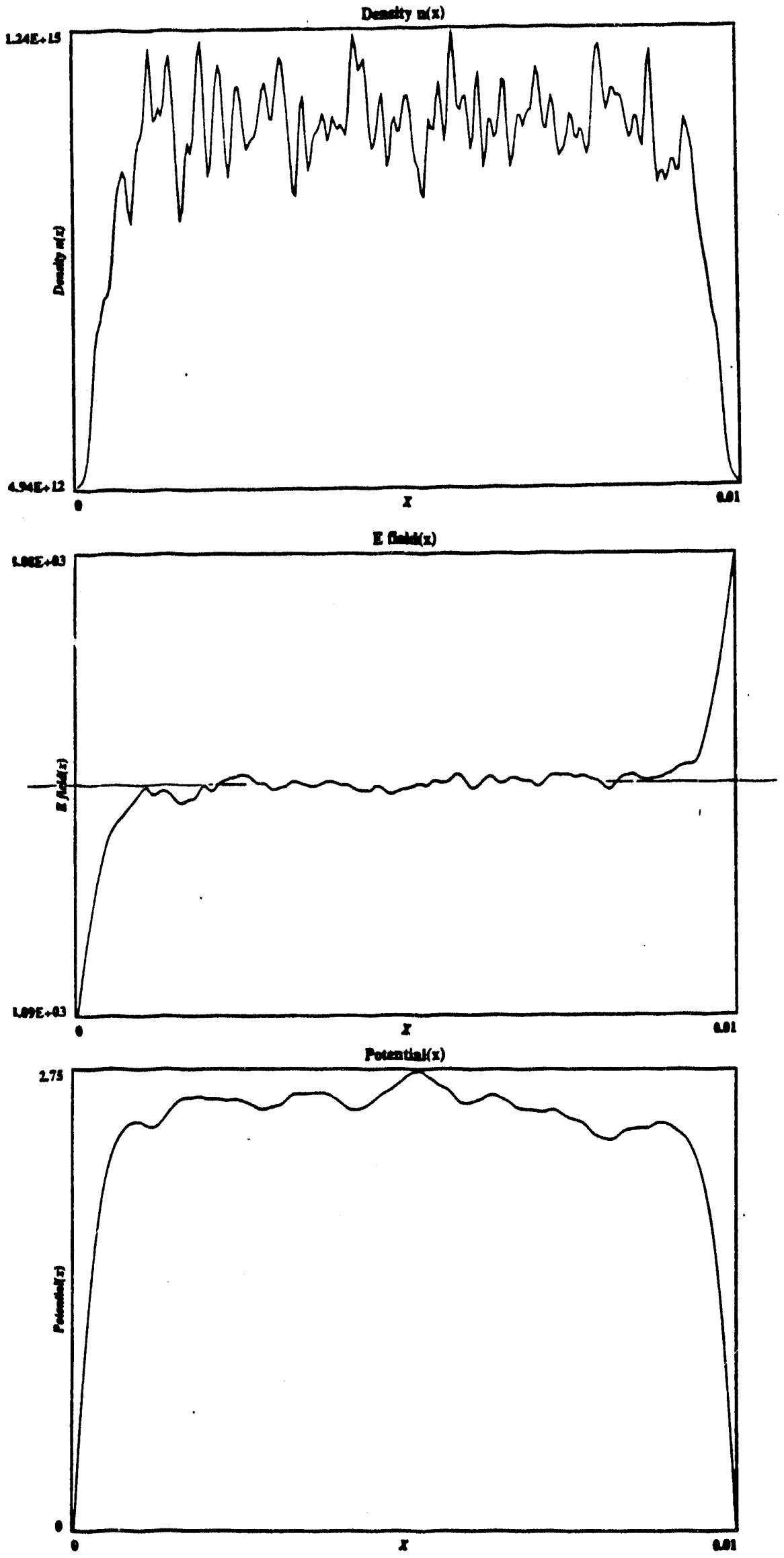

Figure 1: 1d bounded simulation model and result. Fig lc. Snapshot of electron density vs. $x$ at $t>0$. Fig le. Snapshot of electric field vs. $x$, showing the electric field is almost zero in the bulk plasma Fig Id. Snapshot of potential vs. $x$, showing sheaths adjacent to the walls. 


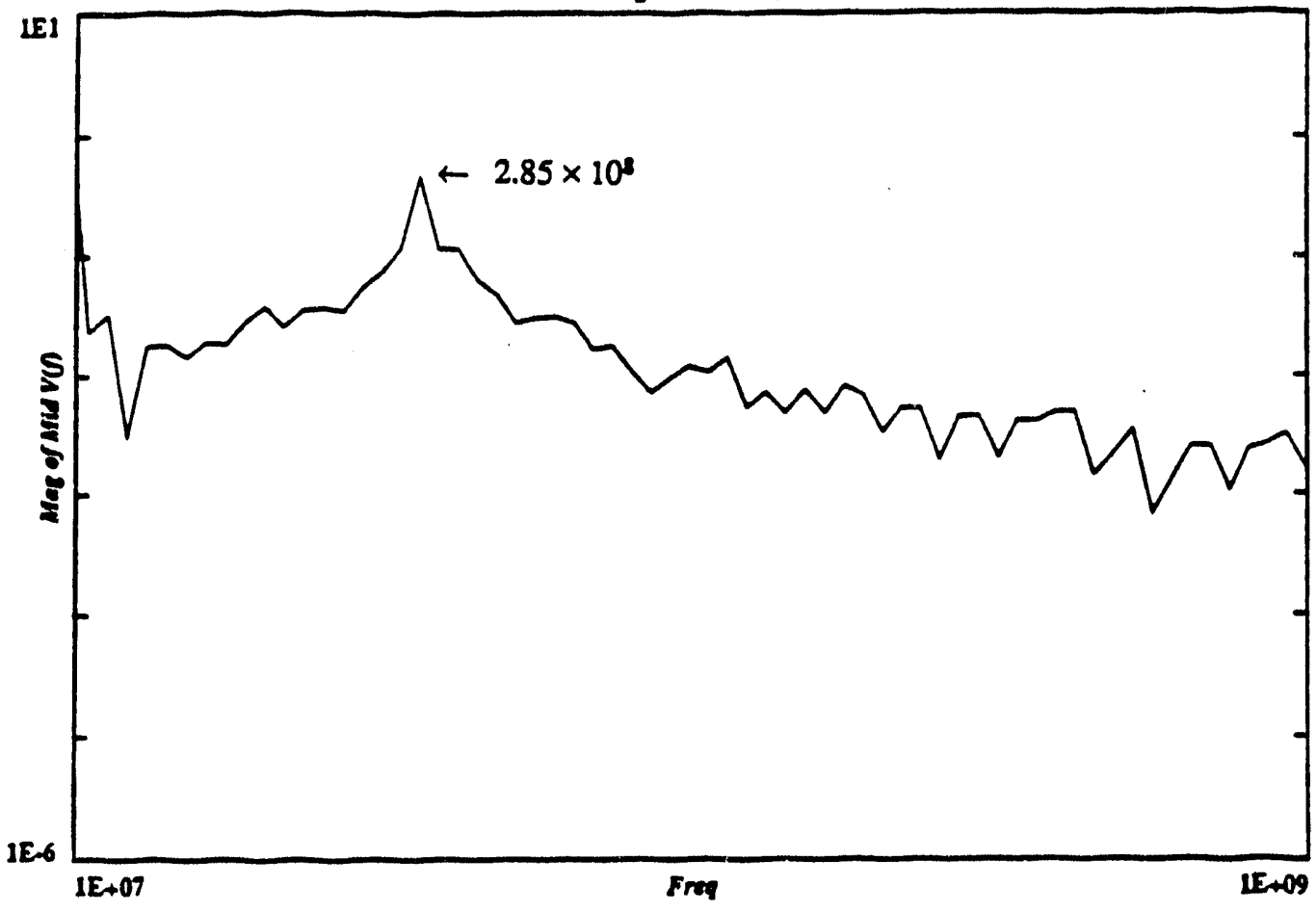

Mas of Curreat(n)

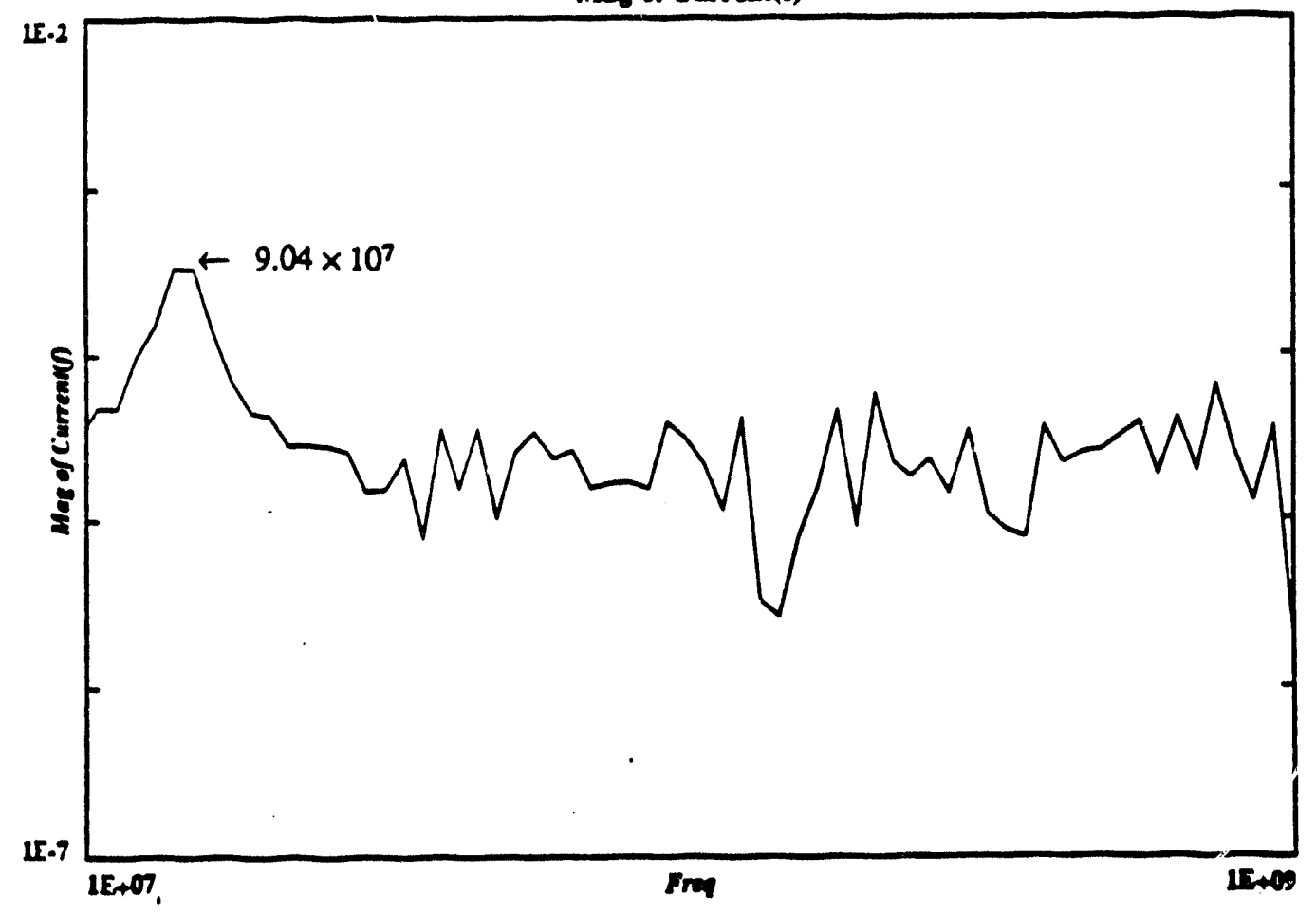

Figure 1: Id bounded simulation model and result. Fig 1g. Frequency spectrum of mid-pocential; using $n=1 \times 10^{15} / m^{3}, f_{p}=285 \times 10^{2}$, ss observed Fig li. Frequency spectrum of exieral current using $\Delta$ from le, gives f arim $=8.94 \times 10^{7}$, close to that observed. 
pre-sheath waves may determine bulk plasma behavior, in other ways yet to be identified.

Sheath waves are the result of charge imbalance when electrons collide with the sheath potential. We bave begun our sheath wave studies by developing a theory for the case of an unmagnetized electrostatic plasma bounded by conducting walls. The sheath waves are assumed to propagate parallel to the walls and the time-average plasma sheath potential drops are perpendicular to the walls. In the plasma bulk, Bohm Gross waves are expected. We have also developed a two dimensional particle simulation that can check our theory. The code is sufficiently general to treat other problems besides unmagnetized sheath waves.

The plan of this paper is as follows. In the second section, we develop a the kinetic theory of sheath waves. In the third section, we describe the simulation model for the unrnagnetized, bounded plasma. In the fourth section, we compare theory and simulation results for waves in the bulk and in the sheath. In the fifth section, we make some concluding remarks.

\section{Kinetic Theory for Unmagnetized Sheath Waves}

We assume a uniform two dimensional plasma of width $L$ bounded by two static sheaths of width $\Delta$ as indicated on Fig. 2. The system is bounded in the $x$ direction by two parallel conducting plates. The static sheaths are modeled as vacuum layers for the electrons, i.e., the electrons are assumed to be reflected on entering the sheath. The ions are treated as immoble with uniform density $n_{0}$, wall to wall. This contrasts with Decyk and Dawson's model (where $\Delta \rightarrow \infty$ ) because although both models have electron reflection, our model has a finite sheath width $\Delta$. It is the consideration of the sheath that also contrasts this model with the Gould-Trivelpiece model for waveguide modes where $\Delta \rightarrow 0$. The sheath waves propagate in the $y$ direction and so the $y$ boundary is taken to be of infinite extent, i.e. periodic over one or more wavelengths.

The linearized kinetic equation for the perturbed electron distribution function $f$, perturbed about a Maxwellian $F_{m}$, and the equation for the perturbed electrostatic potential $\phi^{p}$ in the plasma region may be written

$$
\begin{gathered}
\frac{\partial f}{\partial t}+\vec{v} \cdot \frac{\partial f}{\partial \vec{r}}+\frac{e}{m} \nabla \phi \cdot \frac{\partial F_{m}}{\partial \vec{v}}=0, \\
\nabla^{2} \phi^{P}(x)=4 \pi e \int f d^{3} v,
\end{gathered}
$$

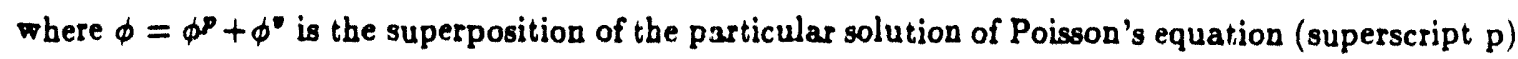
and the homogeneous solution of Laplace's equation (superscript $v$ ). Only the particular solution is given by Eq. (2). The homogenous solution will be similar to the solution of the perturbed potential 


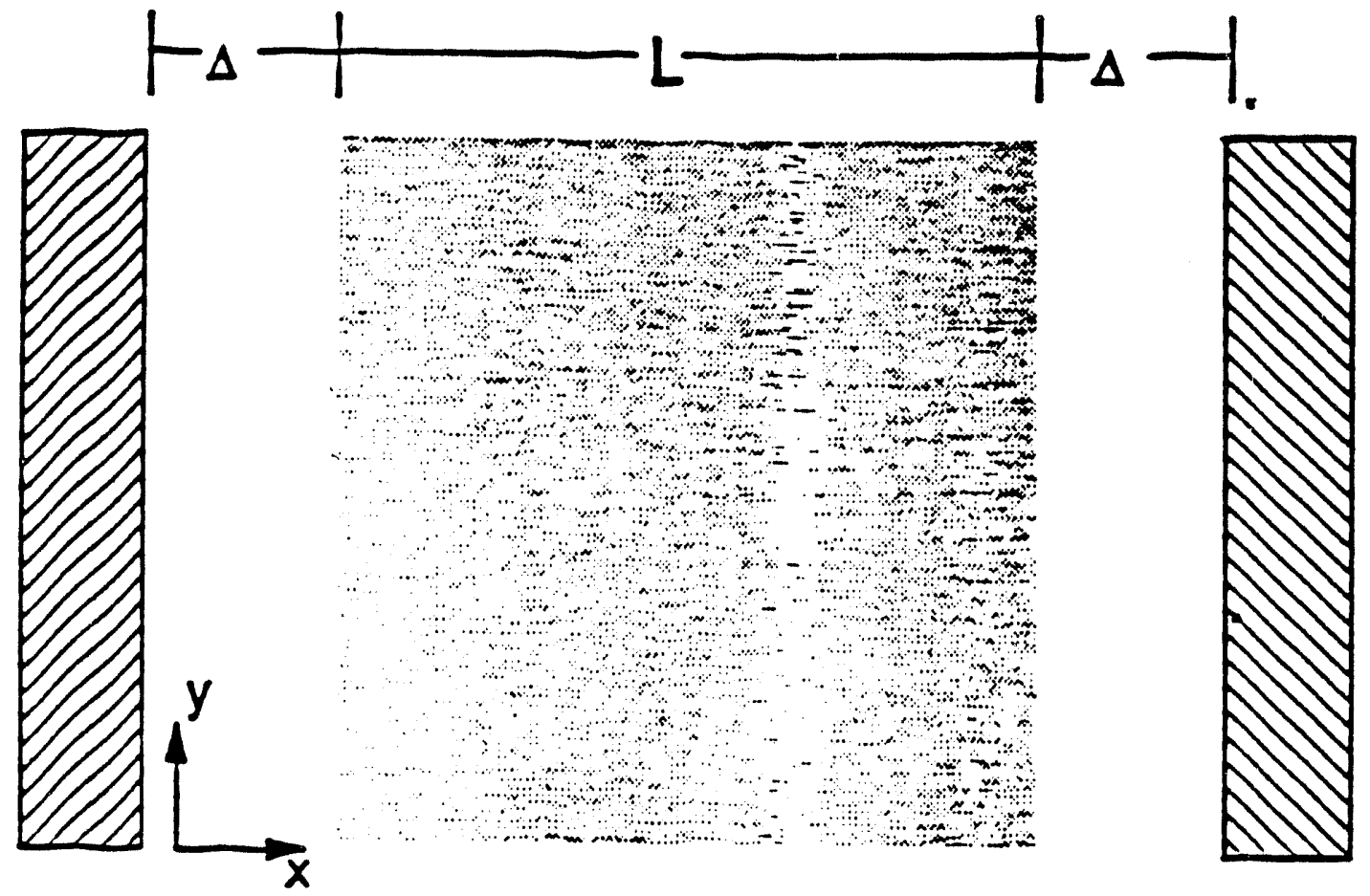

Figure 2: Model for the 2d sheath plasma wave calculations, showing a plasma of length $\mathrm{L}$ bounded by sheaths of width $\Delta$. 
in the sheath region. In the sheath region, the perturbed potential satisfies the equation

$$
\nabla^{2} \phi=0
$$

Assuming an unperturbed electron number density $n_{0}$ only in the plasma region and short circuit boundary conditions, the unperturbed potential takes the usual parabolic form in the ion only sheath regions (called "matrix sheath") and flat in the plasma region. The unperturbed functions are constant in $y$.

Reflecting boundary conditions for the electron distribution function are

$$
\begin{gathered}
f\left(x=\Delta, y, v_{x}, v_{y}, v_{z}\right)=f\left(x=\Delta, y,-v_{x}, v_{y}, v_{z}\right), \\
f\left(x=L+\Delta, y, v_{s}, v_{y}, v_{z}\right)=f\left(x=L+\Delta, y_{y},-v_{x}, v_{y}, v_{z}\right) .
\end{gathered}
$$

Boundary conditions for the electric potential $\phi$ are that $\phi$ and $\partial \phi / \partial x$ must be continuous at $x=\Delta$ and $x=L+\Delta$ and $\phi=0$ at $x=0$ and $x=L+2 \Delta$ for the short circuit boundary condition. Boundary conditions in $y$ are periodic.

To proceed analytically, we assume that any perturbed function $a$ has the form $a(x, y, t)=$ $a(x) e^{i\left(k_{y} y-w t\right)}$. Note that periodicity in $y$ is automatically satisfied by $k_{y}=2 n \pi / L_{y}$. The solution to Eq. (3) for the potential in the sheath region is $\phi=\phi(x) e^{(k, y-w t)}$ where

$$
\phi(x)=A \sinh \left(k_{y} x\right), 0<x<\Delta,
$$

and

$$
\phi(x)=D \sinh \left[k_{y}(L+2 \Delta-x)\right], L+\Delta<x<L+2 \Delta .
$$

Note that these solutions satistfy the short circuit boundary conditions. The homogeneous, or vacuum part, of the potential in the plasma region has a similar form to Eqs. (6) and (7):

$$
\phi^{\prime \prime}(x)=B e^{-k_{y}(s-\Delta)}+C e^{k_{y}(x-L-\Delta)} .
$$

We take the particle reflection conditions, Eqs. (4) and (5), into account by assuming that a particle moves freely along $x$ and by continuing the potential $\phi^{p}(x)$ as an even function beyond the two end surfaces at $x=\Delta$ and $x=L+\Delta$. If a particle is reflected, it would have seen the same potential going from the reflection plane as it would going towards the reflection plane. This is accomplished by continuing $\phi^{\prime}$ as an even function and therefore periodically along the entire $x$ axis. We expand (for the even solutions) the potential $\phi^{p}(x)$ in a Fourier series on the interval ( $\Delta$, $L+\Delta)$

$$
\phi^{\prime}(x)=\sum_{k_{x}=n \pi / L=0}^{\infty} a\left(k_{s}\right) \cos \left(k_{s}(x-\Delta)\right) .
$$


Thus the solution in the plasma region becomes

$$
\phi(x)=B e^{-k_{y}(x-\Delta)}+C e^{k_{y}(x-L-\Delta)}+\sum_{k_{z}=n \pi / L=0}^{\infty} a\left(k_{z}\right) \cos \left(k_{x}(x-\Delta)\right) .
$$

For all sums over $k_{z}$, the $k_{z}=0$ term should be multiplied by 0.5 . Alternatively, we could write $\phi(x)$ in this region as

$$
\phi(x)=\sum_{k_{z}=n=\pi / L=0}^{\infty} \phi\left(k_{z}\right) \cos \left(k_{z}(x-\Delta)\right) .
$$

The relation between $a\left(k_{s}\right)$ and $\phi\left(k_{s}\right)$ is easily shown to be

$$
\phi\left(k_{x}\right)=a\left(k_{x}\right)+\frac{2}{L} \frac{k_{y}}{k^{2}}\left[1-e^{-k_{y} L} \cos \left(k_{x} L\right)\right]\left[B+C \cos \left(k_{x} L\right)\right] .
$$

By applying the technique of integrating along the unperturbed trajectories of Vlasov's equation, Eq. (1), and using the form of $\phi$ given by Eq. (11), the perturbed distribution function $f$ becomes [5]

$$
f(\vec{x}, \vec{v}, t)=-\frac{e F_{m}}{T_{e}} \sum_{k_{z}=n \pi / L=0}^{\infty} \phi\left(k_{x}\right) e^{\left.i\left(k_{z}(z-\Delta)\right)+k_{y} y-\omega t\right)} \frac{k_{x} v_{x} \cos \left(k_{x} L\right)}{k_{x} v_{x}-\omega}
$$

Next, $f(\vec{x}, \vec{v}, t)$ is integrated over velocity space and used in Poisson's equation, Eq. (2), with the form of $\phi^{p}$ given by Eq. (9). The result is

$$
a\left(k_{x}\right)=\phi\left(k_{x}\right)[1-\epsilon(k, \omega)]
$$

where $\epsilon(k, \omega)$ is the dielectric function for an infinite plasma

$$
\epsilon(k, \omega)=1+\omega_{p e}^{2}[1+\zeta Z(\zeta)] / k^{2} v_{T_{e}}^{2}, \zeta=\omega / \sqrt{2} k_{T_{e}}
$$

and $Z(\zeta)$ is plasma dispersion function.

Equation (12) may be used in Eq. (14) to obtain

$$
a\left(k_{x}\right)=(1 / \epsilon(k, \omega)-1) \frac{2}{L} \frac{k_{y}}{k^{2}}\left[1-e^{-k, L} \cos \left(k_{x} L\right)\right]\left[B+C \cos \left(k_{x} L\right)\right] .
$$

Substituing Eq. (16) into Eq. (10), two homogeneous equations for the two unknowns B and C may be obiained by matching the Eq. (10) and Eqs. (6) and (7) at the vacuum-plasma interfaces $x=\Delta$ and $x=L+\Delta$. The boundary conditions are $\phi(x)$ and $\frac{\partial \phi}{\partial x}$ to be continuous, which yields

$$
B= \pm C
$$

and

$$
\begin{gathered}
D(k, \omega)=\tanh \left(k_{y} \Delta\right)+\frac{2 k_{y}}{L} \sum_{\substack{k_{x}=n \pi / L=-\infty \\
n=\text { even, odd }}}^{\infty} \frac{1}{k^{2} \epsilon(k, \omega)}=0 \\
\end{gathered}
$$


where we have used $1 \pm \cos \left(k_{x} L\right)=0,2, \epsilon(k, \omega)$ is an even function of $k_{x}$ and the relation

$$
\sum_{\substack{k_{x}=n \pi / L=-\infty \\
n=\text { even, odd }}} \frac{1}{k_{x}^{2}+k_{y}^{2}}=\left\{\begin{array}{ll}
\frac{L}{2 k_{y}} \operatorname{coth}\left(\frac{k_{y} L}{2}\right), & \text { even } \\
\frac{L}{2 k_{y}} \tanh \left(\frac{k_{y} L}{2}\right), & \text { odd }
\end{array}\right\} .
$$

The upper sign in Eq. (17) corresponds to modes which are symmetric about the midplane of the slab. For this mode only even values of $n$ appear in the sum in Eq. (18). The lower sign in Eq. (17) corresponds to asymmetric mode and for it only odd values of $n$ appear in the sum in Eq. (18).

Equation (18) is a general formula for the dispersion relation of the plasma oscillation in the presence of two conducting walls. We seek a complex frequency solution, $\omega=\omega\left(k_{y}\right)+i \gamma\left(k_{y}\right)$ of Eq. (18) first in the long-wavelength limit so that $k_{y} \lambda_{D_{e}} \ll 1$. In this limit, the plasma dispersion function reduces to

$$
\epsilon(k, \omega)=1-\frac{\omega_{p e}^{2}}{\omega^{2}}-\frac{3}{2} \frac{\omega_{p e}^{2} k^{2} v_{T e}^{2}}{\omega^{4}}+i \sqrt{\pi} \frac{2 \omega_{p e}^{2} \omega}{k^{3} v_{T e}^{3}} e^{-\frac{\omega^{2}}{k_{v}^{2} \cdot v_{T e}^{2}}}
$$

Let us look first at $\omega<\omega_{\text {pe }}$, from Eqs. (18) and (19), we obtain

$$
D\left(k_{y}, \omega\right)=\tanh \left(k_{y} \Delta\right)+\frac{1}{1-\frac{\omega_{y:}^{2}}{\omega^{2}}}\left\{\begin{array}{l}
\operatorname{coth}\left(\frac{k_{y} L}{2}\right)-\frac{k_{y}}{r} \operatorname{coth}\left(\frac{k_{y} r}{2}\right) \\
\tanh \left(\frac{k_{y} L}{2}\right)-\frac{k_{y}}{r} \tanh \left(\frac{k_{y} r}{2}\right)
\end{array}\right\}=0
$$

where

$$
\tau^{2}=k_{y}^{2}+\frac{2}{3} \frac{\omega^{2}}{\omega_{p e}^{2} v_{T e}^{2}}\left(\omega_{p e}^{2}-\omega^{2}\right)
$$

Comparing the sheath wave with the surface wave occuring at a vacuum-plasma interface, (i. e., $\Delta \rightarrow \infty)$, the main difference is that vacuum dielectric constant $\epsilon_{0}$ is $\operatorname{coth}\left(k_{y} \Delta\right)$ in the sheath wave case instead of $\epsilon_{0}=1$. Furthermore, Ref. [4] uses a warm electron fluid model of the plasma while we use a fully kinetic model. This results in differences in the last terms of Eqs. (21) and (22) when compared to similar equations in Ref. [4]. From Eq. (21) we obtain the following dispersion relation:

$$
\omega=\frac{\omega_{p e}}{\sqrt{1+\operatorname{coth}\left(\frac{k_{y} L}{2}\right) \operatorname{coth}\left(k_{y} \Delta\right)}}\left(1+\frac{\sqrt{3}}{2} k_{y} \lambda_{D_{e}} \sqrt{\tanh \left(k_{y} \Delta\right) \tanh \left(\frac{k_{y} L}{2}\right)}\right) .
$$

for the symmetric sheath wave modes and

$$
\dot{\omega}=\frac{\omega_{p e}}{\sqrt{1+\tanh \left(\frac{k_{y} L}{2}\right) \operatorname{coth}\left(k_{y} \Delta\right)}}\left(1+\frac{\sqrt{3}}{2} k_{y} \lambda_{D_{e}} \sqrt{\tanh \left(k_{y} \Delta\right) \operatorname{coth}\left(\frac{k_{y} L}{2}\right)}\right) .
$$

for the asymmetric sheath wave modes. Eqs. (23) and (24) have been plotted on Fig. 3 as a solid curve and a dotted curve, respectively, for $L=38 \lambda_{D e}$ and $\Delta=2 \lambda_{D e}$. 


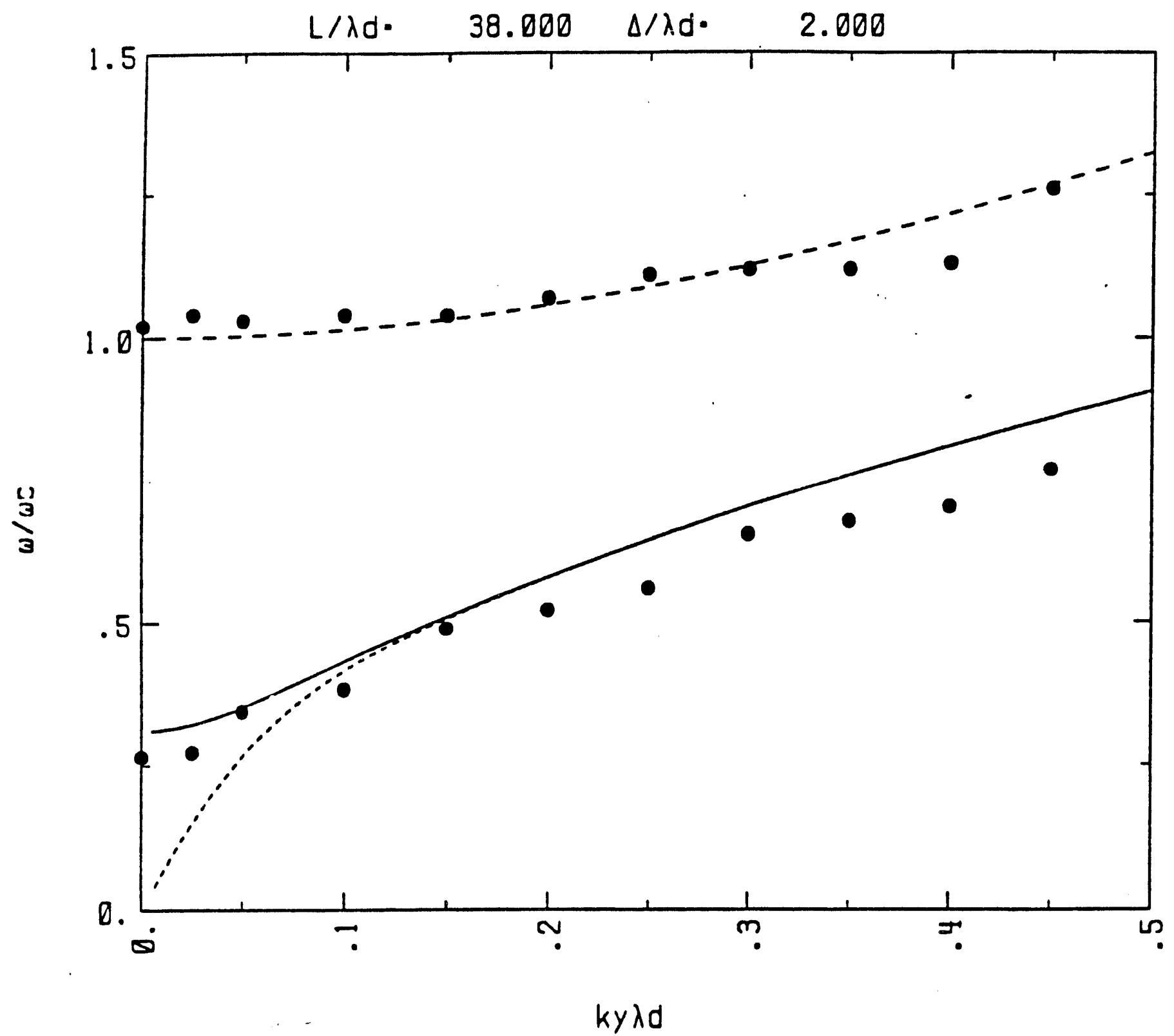

Figure 3: Disperson relations for electron waves calculated from kinetic theory and the simulation results. Bohm-Gross waves are shown as a dashed curve $\left(k_{z}=0\right)$, top, asymmetric sheath waves as a solid curve, symmetric sheath waves as a dotted curve. The simulation results are dots. The BohmGross waves were measured at $x=L_{x} / 2$. The sheath waves were measured at $x=2 \lambda_{D_{\text {a }}} .\left(M_{i} \rightarrow \infty\right.$, $L_{\mathrm{x}} / \lambda_{D_{0}}=42,64 \times 64$ grid, $L=38 \lambda_{D_{e}}, \Delta=2 \lambda_{D_{0}}$ and 10000 particles). The smallest $k_{y} \lambda_{D}$ is 0.02 , which also is $k_{5} L_{2} / 2=0.42$. 
In the limit $k_{y} \Delta>1$ and $k_{y} L \gg>1$, both symmetric and asymmetric modes merge and the semi-infinite vacuum-plasma (or isolated slab) electron surface wave results are recovered [8]:

$$
\omega=\frac{\omega_{p e}}{\sqrt{2}}\left(1+\frac{\sqrt{3}}{2} k_{y} \lambda_{D_{e}}\right) .
$$

We find that the collisionless damping of the sheath waves in the limit $k_{y} L \gg 1$ is the same as the semi-infinite vacuum case [5]:

$$
\gamma_{0}=\omega_{p e} \sqrt{\frac{2}{\pi}} k_{y} \lambda_{D_{e}}
$$

In the opposite limit, $k, \Delta \ll 1$ and $k_{y} L \ll 1$, we find from Eq. (23) and (24) that the symmetric mode frequency goes to zeto and asymmetric mode frequency becomes the plasma series resonance[2]

$$
\omega=\frac{\omega_{p e}}{\sqrt{1+\frac{L}{2 \Delta}}}=\omega_{p e} \sqrt{\frac{2 \Delta}{L_{s}}} .
$$

(The series resonance was observed in 1d simulations shown earlier in Figure 1.)

In the cold plasma approximation, comparing sheath waves in a planar system with GouldTrivelpiece (G-T) waves in a plasma waveguide, we find that both waves are generally the same. For both symmetric sheath waves in a slab and circularly symmetric G-T waves in a pipe, wave propagation occurs from $\omega=0$ to $\omega=\frac{w_{x}}{\sqrt{2}}$. Asymmetric sheath waves in a slab and G-T waves in a pipe with an $m=1$ mode angular variation both have the same high frequency limit $\omega=\frac{\omega_{x}}{\sqrt{2}}$ and exhibit a lower cutoff frequency. The lower frequency limit of G-T waves is [3]

$$
\omega=\frac{\omega_{p e}}{\sqrt{1+\frac{b^{2}+a^{2}}{b^{2}-a^{2}}}}
$$

where $a$ and $b$ are the radii of the plasma column and the conducting cylinder, respectively. The lower frequency limit of sheath waves in a slab is given by Eq. (27). The lower frequency limit of sheath waves in a slab is much lower than $\omega_{\text {pe }}$ since generally $\Delta \ll L$. The lower frequency limit of G-T waves is much lower than $\omega_{p e}$ only if $a \approx b$. It is worth noting that $\Delta$ for the sheath waves is self consistently determined by plasma properties and wall conditions at equilibrium and that electron reflection boundary conditions are satisfied by equilibrium sheath potential.

Equations (18), (20) and (22) lead to the frequency and the spatial damping for the sheath waves because $\tau^{2}$ in Eq. (22) may be positive. Equations (18), (20), and (22) lead to the frequency and the $x$ wavenumber $k_{z}$ for bulk waves because $\tau^{2}$ may be negative. Thus, if $\omega>\omega_{\text {pe }}, E q$. (20) gives the usual Bohm-Gross plasma waves which have also been plotted on Fig. 3 as the dashed curve (for $k_{x}=0$ ). The propagating bulk waves satisfy the usual infinite plasma dispersion relation

$$
\omega^{2}=\omega_{p e}^{2}+\frac{3}{2}\left(k_{y}^{2}+k_{x}^{2}\right) v_{T e}^{2}
$$


where $k_{x}=\sqrt{-\tau^{2}}$ is determined by Eqs.(18), (20) and (22) as

$$
\tan \left(\frac{k_{x} L}{2}\right)=\frac{k_{x}}{k_{y}}\left\{\tanh \left(k_{y} \Delta\right) \frac{\sqrt{1+12\left(k_{y}^{2}+k_{z}^{2}\right) \lambda_{d e}^{2}}-1}{\sqrt{1+12\left(k_{y}^{2}+k_{z}^{2}\right) \lambda_{d e}^{2}}+1}+\tanh \left(\frac{k_{y} L}{2}\right)\right\},
$$

It is clear from inspection that this equation has an infinite number of roots $k_{z}$. The spacing between them is about $\frac{2 \pi}{L}$. In the $1 \mathrm{~d}$ model, $k_{y}=0$, Eqs. (29) and (30) reduce to standing Bohm-Gross waves.

\section{Simulation Model for IJnmagnetized Sheath Waves}

Particle simulation is used to model the plasma because kinetic effects are accounted for naturally. In particle simulation, superparticles, each representing a large number of actual plasma particles, are muved in phase space according to particle equations of motion. The particles are used to calculate charge and current densities which are source terms in Maxwell's equations and the resulting fields are self consistently used in the particle equations of motion.

Sheath and butk waves are modeled in by placing superparticle electrons, each representing a large number of actual electrons, and a uniform ion background with density $n_{0}$ between two shorted conducting walls (wall to wall). The electrons are initially uniform in space with density $n_{0}$ and Maxwellian in velocity with temperature $T_{e}$. The computational cycle is as follows:

- Given the field on a numerical grid in space, the superparticle electrons are advanced in phase space by the usual leap frog numerical form of the equations of motion. Linear weightirg is used to determine the electric field at each electron.

- The electrons that actually go into the wall are deleted from the list of superparticles and the change in wall charge is calculated. Note that with a conducting wall, wall charge is uniform in $y$, but the induced surface charge density is not, due to the non-uniform distribution in $y$ of the charge density, inside the plasma.

- The electrons that remain in the region between the two walls are used to compute the electron charge density at each grid point using linear weighting.

- The total charge density, electrons and background, is then used as the RHS of Poisson's equation. The equation is solved with the boundary conditions periodic in $y$ and $\phi=0$ at the conducting walls.

- The electric field is calculated form the potential using the finite difference version of $\vec{E}=-\nabla \phi$ except at the walls where Gauss' Law is used to determine $\vec{E}$. Then, the cycle repeats. 

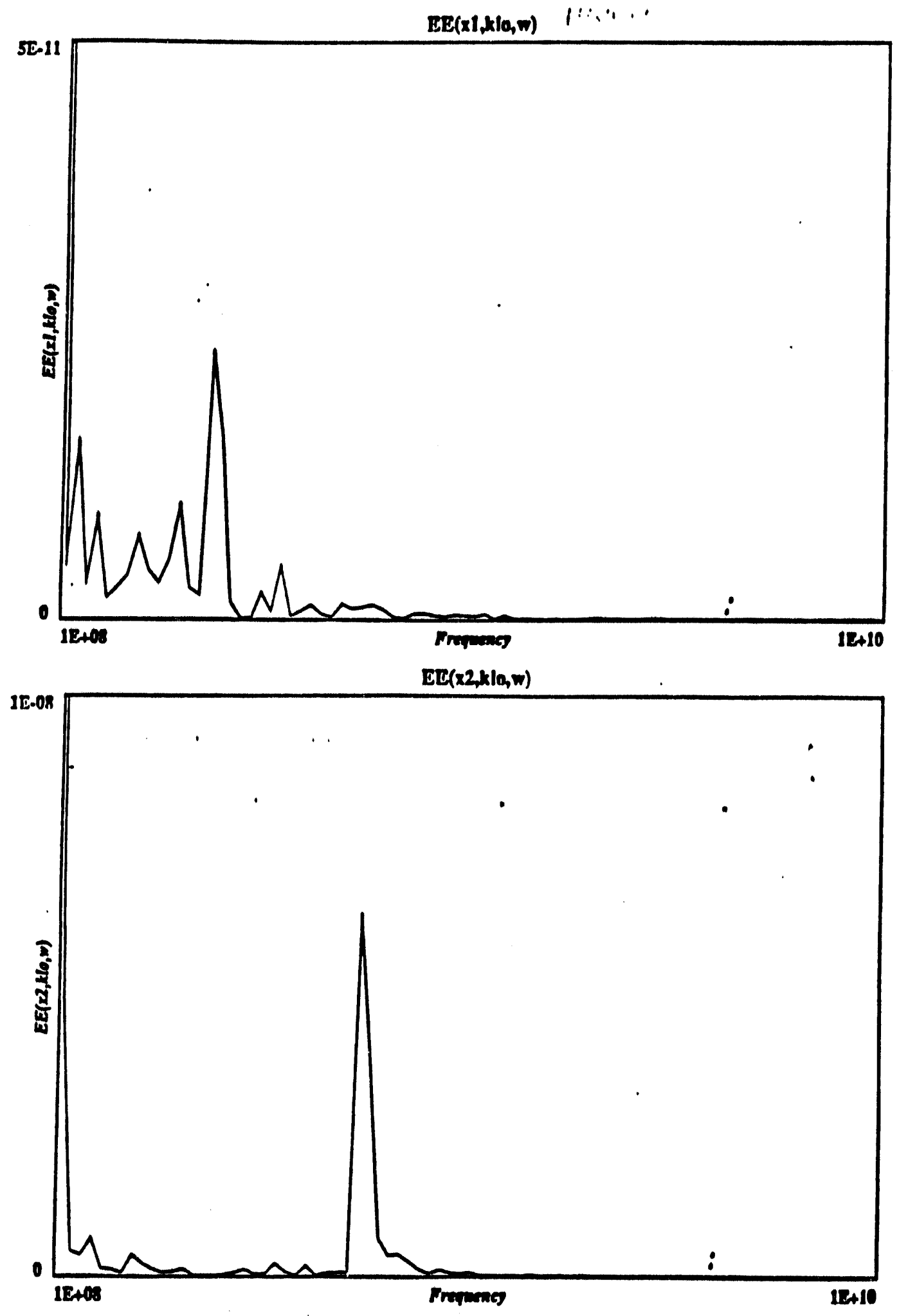

Figure 4(a): The power spectrum for a $2 d$ particle simulation of a aheath wave; for the $k_{y} \lambda_{D_{0}}=$ 0.15 simulation, measured at $x=1.5 \lambda_{D}$ for sheath waves; (b) the anme for Bohm-Groes waves, measured at $x=L_{s} / 2$. 
Because the fields and potential are taken to be periodic in the $y$ direction, it is possible to solve Poisson's equation using fast Fourier transforms in the $y$ direction to speed up computation and give spectral diagnostics[10]. The charge density is transformed in the $y$ direction for every $\boldsymbol{x}$ coordinate. Then, only a one dimensional tridiagonal matrix inversion is required to solve the transformed form of the Poisson equation for each $k_{y}$ mode. Boundary conditions are accounted for in the $k_{y}=0$ mode. Finally, an inverse transiorm is done for every $x$ coordinate to give the potential on the $x, y$ grid.

The above algorithm is time explicit which means the highest physical frequency in the system must be resolved by the numerical method or numerical instability will result. For this case, the highest frequency is near the electron plasma frequency giving the constraint $\omega_{\text {pe }} \Delta t<2$, where $\Delta t$ is the simulation time step.

It was previously mentioned that each superparticle represents a large number of actual particles. For good statistics, the superparticle should represent the smallest number of actual particles with in the limits of computational feasibility. For example, if the actual system contains $10^{10}$ particles, then for 10000 superparticles, each superparticle represents $10^{6}$ real particles. To get better representation, one may want to go to $\mathbf{4 0 0 0 0}$ superparticles but this may be prohibitive in term of CPU charges. The majority of CPU time is taken up in particle pushing and weighting. The time taken to solve Poisson's equation and compute diagnostics is relatively trivial.

The code PDP2 was used to simulate the plasma. It is similar to one dimensional plasma simulation codes [1] but with the $y$ periodic dimension added. PDP2 may be exeruted in a window environment such as XGRAFIX on workstations. This allows the viewing of phystcs as it bappens.

\section{Comparison of Simulation and Theory for Sheath Waves}

\subsection{The first results with a random number generator for particle loading}

Recults were computed for the initial parameters of electron density $n_{0}=1 \times 10^{15} / \mathrm{m}^{3}, T_{e}=1 \mathrm{eV}$, and a system length $L_{\varepsilon}=L+2 \Delta=0.01 \mathrm{~m}$, i.e., $42 \lambda_{D_{e}}$, between the walls. For these parameters, the measured sheath width was $2.0 \lambda_{D_{e}} . K_{y} \lambda_{D_{e}}$ was varied by changing the input parameter $L_{y}$ since $k_{y}=2 \pi / L_{y}$. (We only measure the longest wavelength mode as it has the least noise.) A $64 \times 64$ grid was used to resolve $\lambda_{D_{e}}$. A time step size was used such that $\omega_{\text {pe }} \Delta t=0.0892$. For each simulation, 10000 superparticle electrons were used so that there were 2.5 superparticles per cell at $t=0$. Real electron masses were used. The ions are uniform and immobile, at density $n_{0}=1 \times 10^{15} / \mathrm{m}^{3}$.

To compare with theory, $\phi$ was Fourier transformed in $y$ for a specific location in $x$, either in 
the sheath to measure sheath waves, or in the bulk to measure bulk plasma waves. The magnitude $\phi\left(k_{y}\right)^{*} \phi\left(k_{y}\right)$ produces a signal in time which can be Fourier transformed to determine frequency. Peaks that appear on the frequency spectrum are located at twice the actual frequency since a transform of a quantity similar to field energy is done. Figure 3 gives the comparison of theory and simulation for various values of $k_{y} \lambda_{D_{e}}$, where the dots are the particle simulation results. Since there is an uncertainty in the location of the sheath-plasma interface, we have measured the sheath waves at $x=2 \lambda_{D}$ and at $x=1.5 \lambda_{D_{e}}$ (Fig. 3 ) in the sheath with identical results. The comparison is quite good for both the bulk Bohm Gross waves (upper branch) and the asymmetric sheath waves (lower branch) independent of where the measurement is taken. The simulations show a tendency to favor the asymmetric sheath waves. We have no points for the symmetric sheath wave for small $k_{y}$. For the bulk waves, we took $k_{z}=0$ (effectively one dimensional) in Eq. (29) to draw the analytic curve in Fig. 3. This was done because we measured waves in the simulation at a specific location in $x$ thus throwing out any information about the particular $k_{x}$. In principal, one could instead take Fourier tranoforms in $x$ and $y$ and keep track of a particular $k_{z}, k_{y}$ pair in time to measure the corresponding $\omega$ for comparison to Eq. (29).

Figure 3 gives the frequency spectrum for sheath and bulk modes respectively. The sheath wave measurements were taken at $x:=1.5 \lambda_{D_{e}}$ for the $k_{y} \lambda_{D_{e}}=0.15$ run. Figure 5 is a snapshot of $\phi$ contours and $\phi$ versus $x, y$ at a specific time for the $k_{y} \lambda_{D_{e}}=0.15$ run. To check the simulation accuracy, the simulation for $k_{y} \lambda_{D_{e}}=0.1$ was run with 40000 superparticle electrons. The same frequencies for the sheath and bulk modes were recovered.

Our simulations indicate that the electron density is non zero in the sheath region as shown on Fig. 1c. Our analytic model requires a zero electron density in the sheath. Because of the inhomogeneity of the electron density can any new modes of surface oscillations arise? A previous surface wave study has shown that if the function of electron density with respect to the spatial variable ( $x$ in our case) is monotone, only the known surface waves of the form given by Eq. (25) exist [12]. The monotone density variation is consistent with our simulation results. Furthermore, simulation results also indicated that the monotone density variation does not effect our analytic model. Therefore, it would seem that not only Eq. (25) is valid, but also our entire theory is valid. If the function of density has as extremum, different potential surface modes can also exist [12].

\subsection{The recent results with a quiet start (particle loading) and discussions}

The results presented in Section 4.1 were obtained over a year ago, and appear to fit much of the predicted dispersion. However, we took a closer look and discovered that we did not have the well known Gould-Trivelpiece waves for a warm, hounded, unmagnetized plasma; such could 


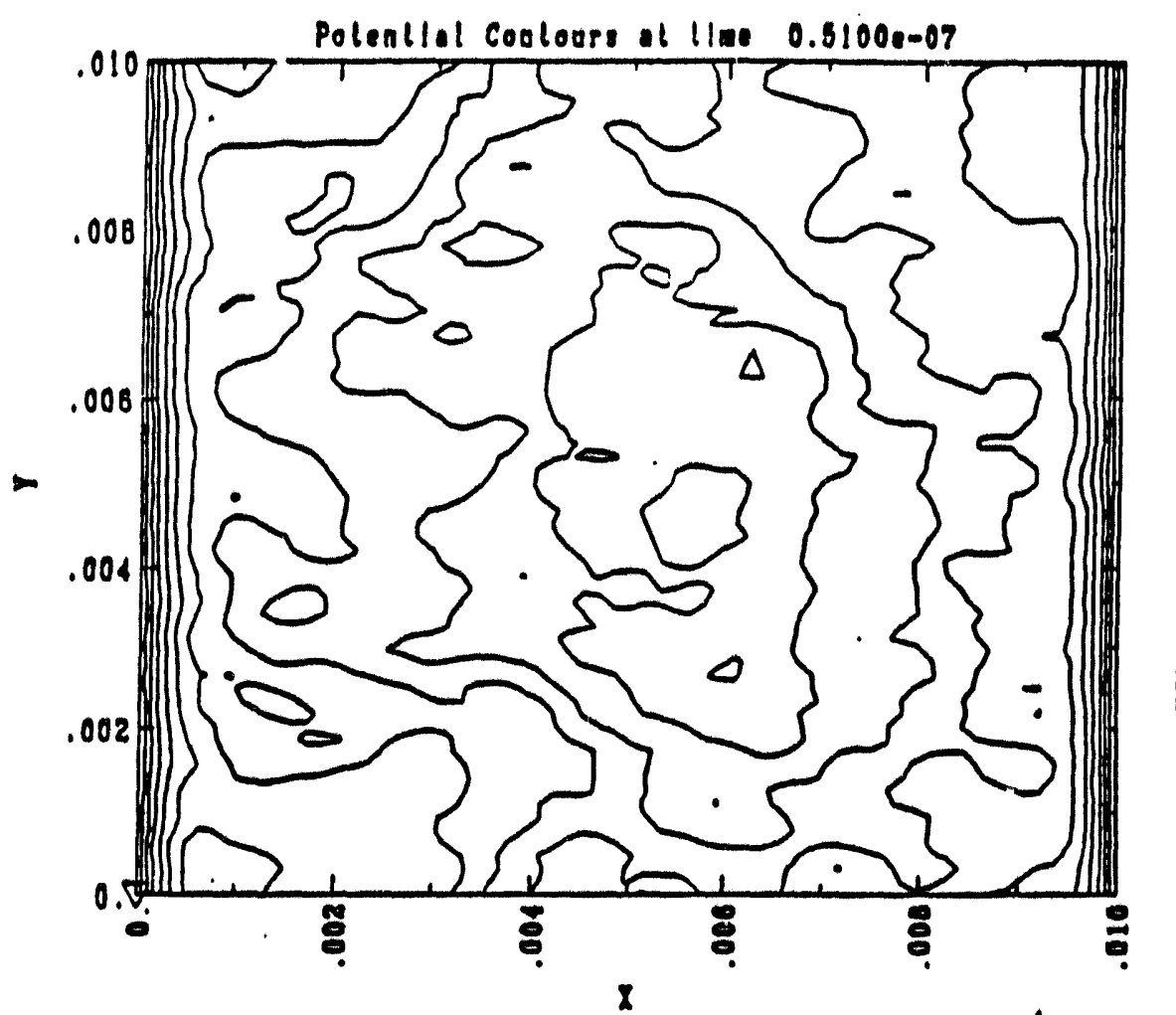

10

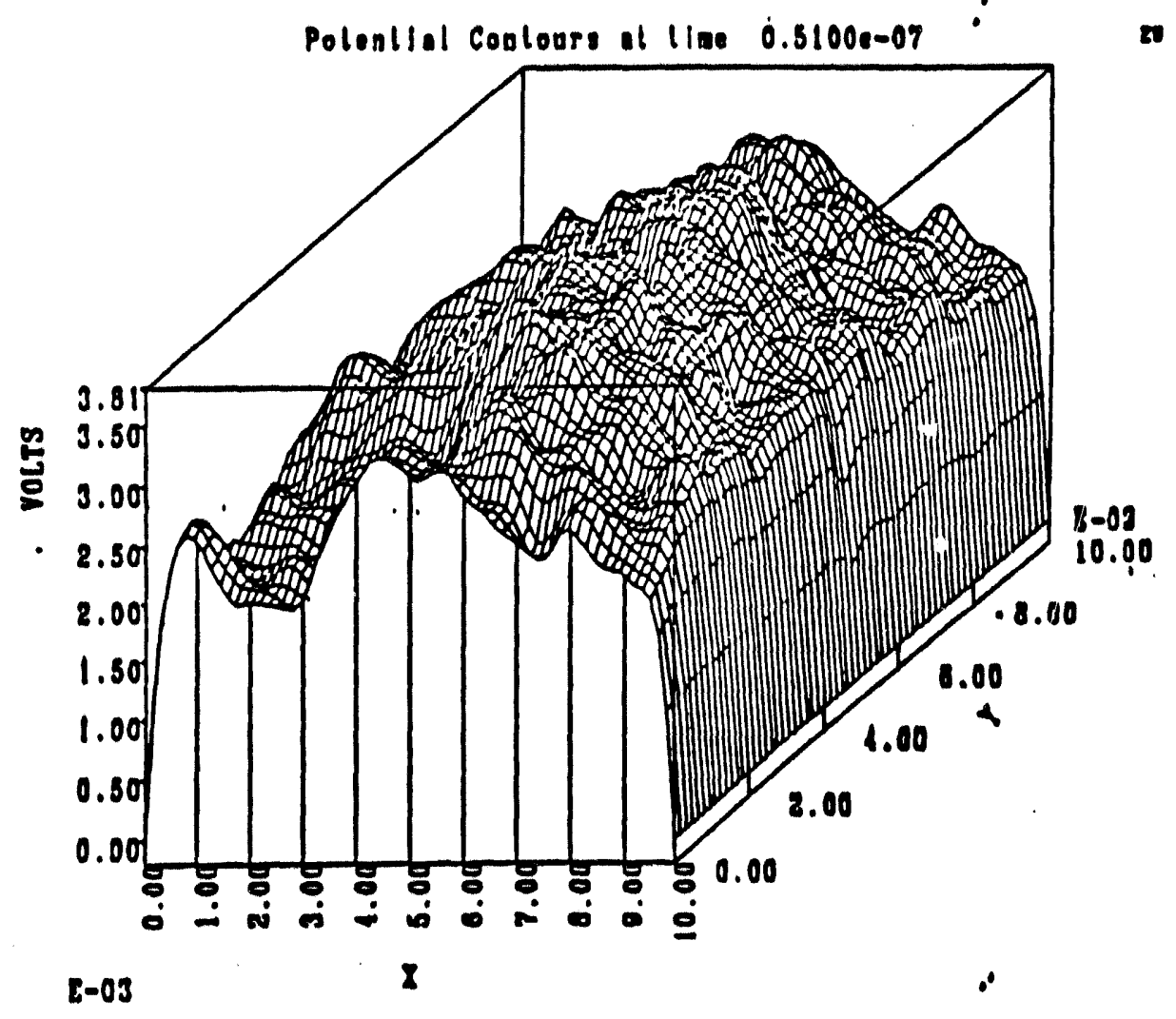

Figure 3a and b: A snapshot of $\phi(x, y)$ contours and 3d plot of $\phi(x, y)$ at a specific time for $k_{y} \lambda_{D_{e}}=0.15$. 
be the low frequency symmetric mode branch in the theory, but no frequencies were obtained for these wavenumbers. Also, while the main dipole resonance was found (the asymmetric mode, or series resonance), the additional dipole resonances (Tonke-Dattner or Parker-Nickel-Gould) were not found. Some new scaling was tried, conjirming what was already obtained.

Looking closer, we found that the presumed quiet start (particle loading at $t=0$ ) was not as quiet what we wanted, that a quieter start was possible, which was implemented. We also found that our use of the IFT for obtaining the power spectra for, say, potential at a given $x, y$ or at a given $x$ for one value of $k_{y}$, was not as good as we could make it, so that was improved. The next step was to try repeat the first results of 1991 with a random number generator; this we were unable io do in detail. Bence, the 1991 results, while presented in Section 4. 1, are given, but not confirmed.

With the improved version of PDP2, we tried a number of excitations in order to obtain the dispersion. We were becoming more and more uneasy that there may not a simple frequency. wavenumber dispersion plot for this model, which, after all, is an inhomogenous medium, with zero-order density a function of $x$. That is, in the simplest way of thinking, there may be waves associated with bulk plasma (which cannot be found if our "probes" are in the sheath), and vice versa, waves localized in the sheath (which may not be found with our "probes" are in the bulk plasma). No matter, as far as the validity of the simulation is concerned; what waves there are will show up and it is up to us identify them.

We tried several approaches, much as if we were doing a laboratory experiment. We will give these results briefly, as they were done in roughly one day (with quite a few days of run-up tries).

First, we drove the plasma with a potential varing at 64 frequencies, at two $x, y$ locations, one near the sheath edge and one in the bulk. We then looked at the potential downstream, at the same $x$ 's, but about $L_{y} / 2$ away, expecting a signal to reach there about $T=L_{y} /\left(2 v_{T_{0}}\right)$, where we guesed that the grcup velocity might be on the order of $v_{T}$. . We made copies of the time records of the exciting and received potentials, and compared them, looking for a possible phase shift, or time delay, due to a finite propagation time. We could "see" (or imagine) such, but decided that we would need to work out a detailed correlation technique in two dimensions in order to make the measurements acceptable. (There have been some very good plasma probing techniques done lately on such; we merely need to copy these.)

Second, we tried pulsing on and off a DC potential, with the same probes. We pick up no obvious reception of the pulse at some time $T$ later (except for a prompt reception, taken to be the vacuum response, due to solving Poisson's equation and not the full E\&M Maxwell set), as shown in Fig. 6. We repeated the same, with a pulse in the charge density, which worked somewhat better, but with 


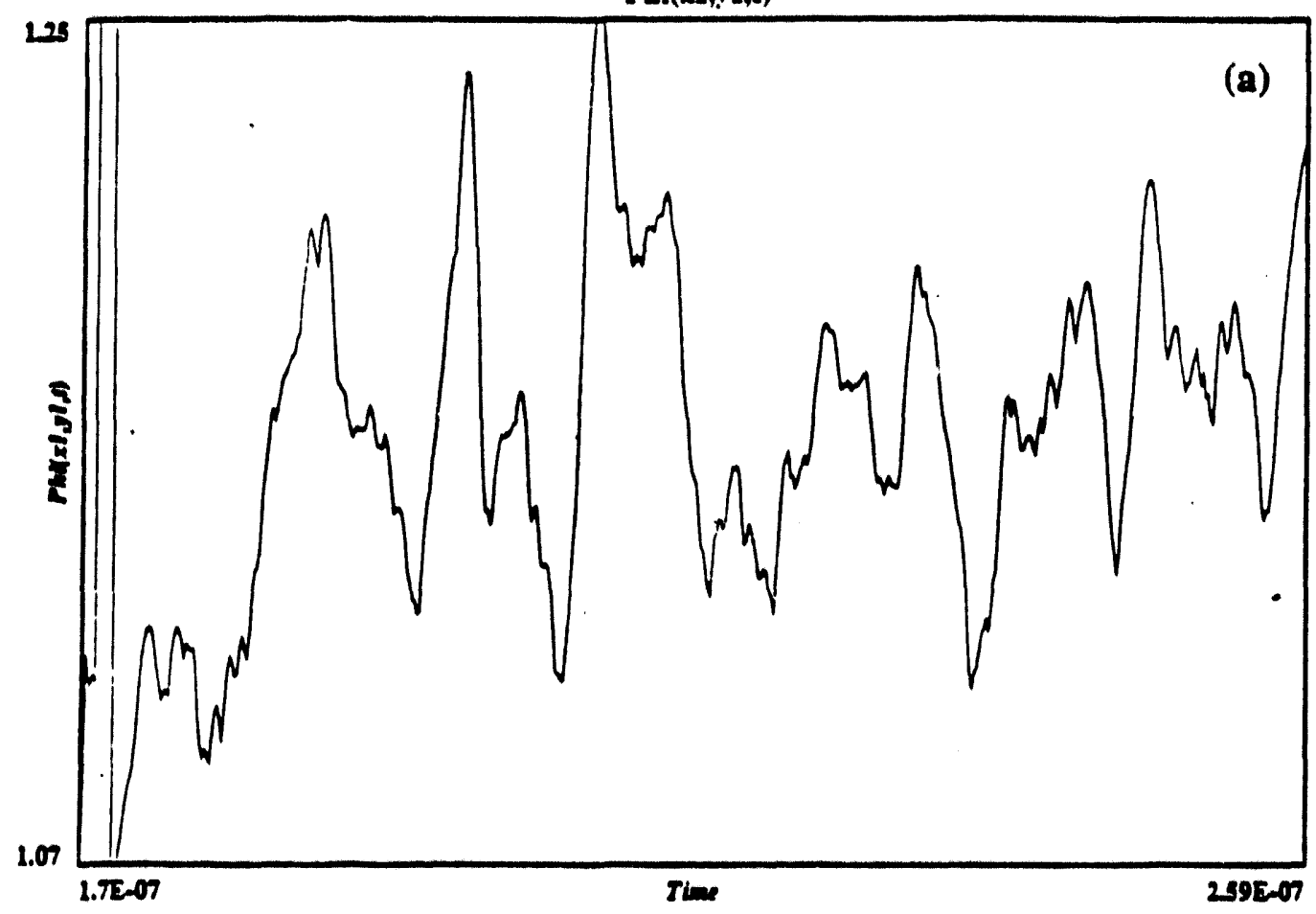

$\operatorname{Pui}(\mathbf{x}, \mathbf{2} \mathbf{2})$

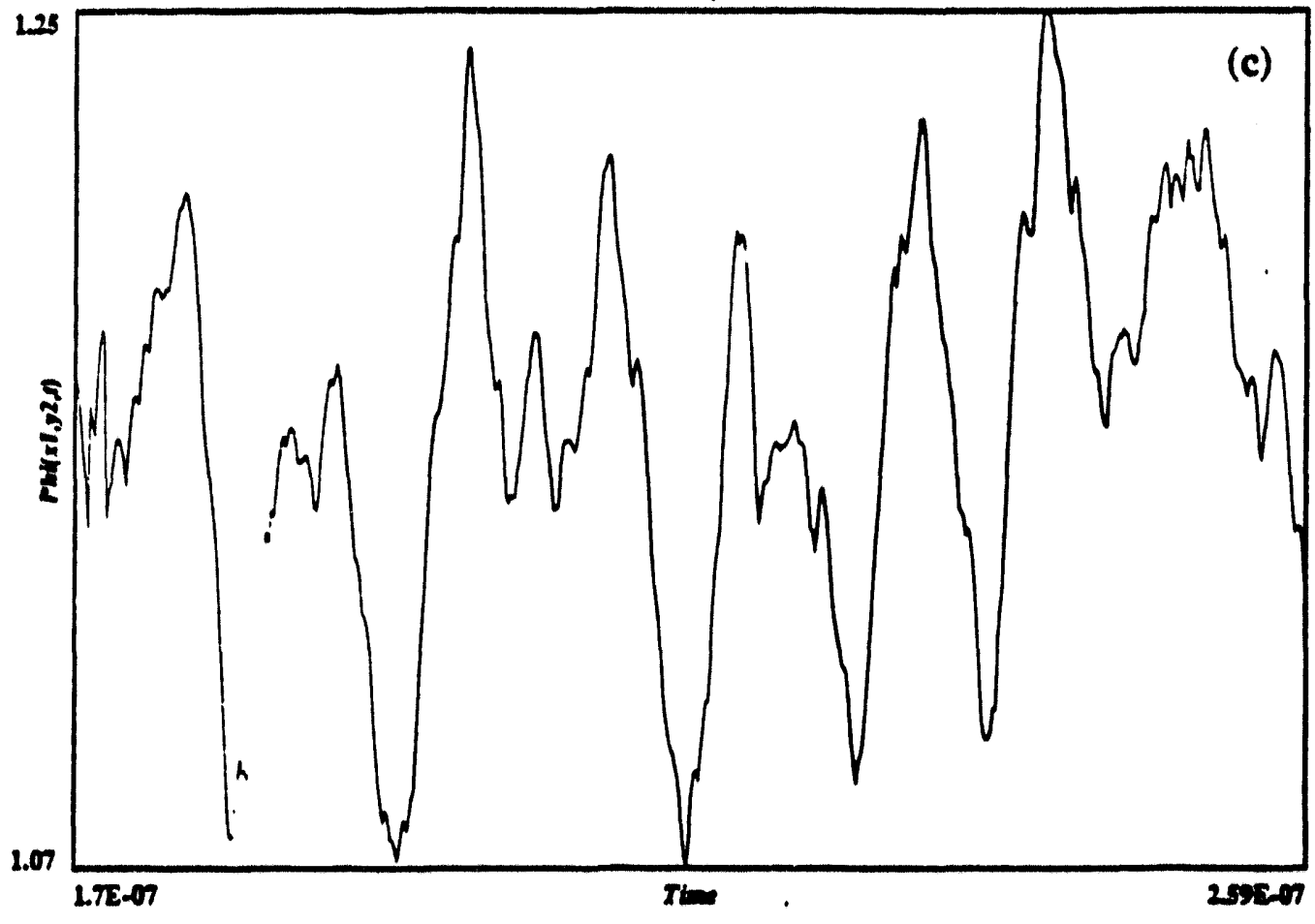

Figure 6: The time records of a pulsing on and of a DC potential an two locations. Fig 62. Source $x_{1}$, $y_{1}$. Fig $6 c$. Receiving them at $x_{1} y_{2}$. Here $x_{1}=7 \Delta x$ (heath edge) and $x_{2}=30 \Delta x$ (bulle plasma), $y_{1}-y_{2}=L_{1} / 2$. The pulse was on for $5 \Delta$, as seen dear $t=1.7 e-7$ in $a$ and $b$. 
$\operatorname{Pan}(x 2, y 1)$

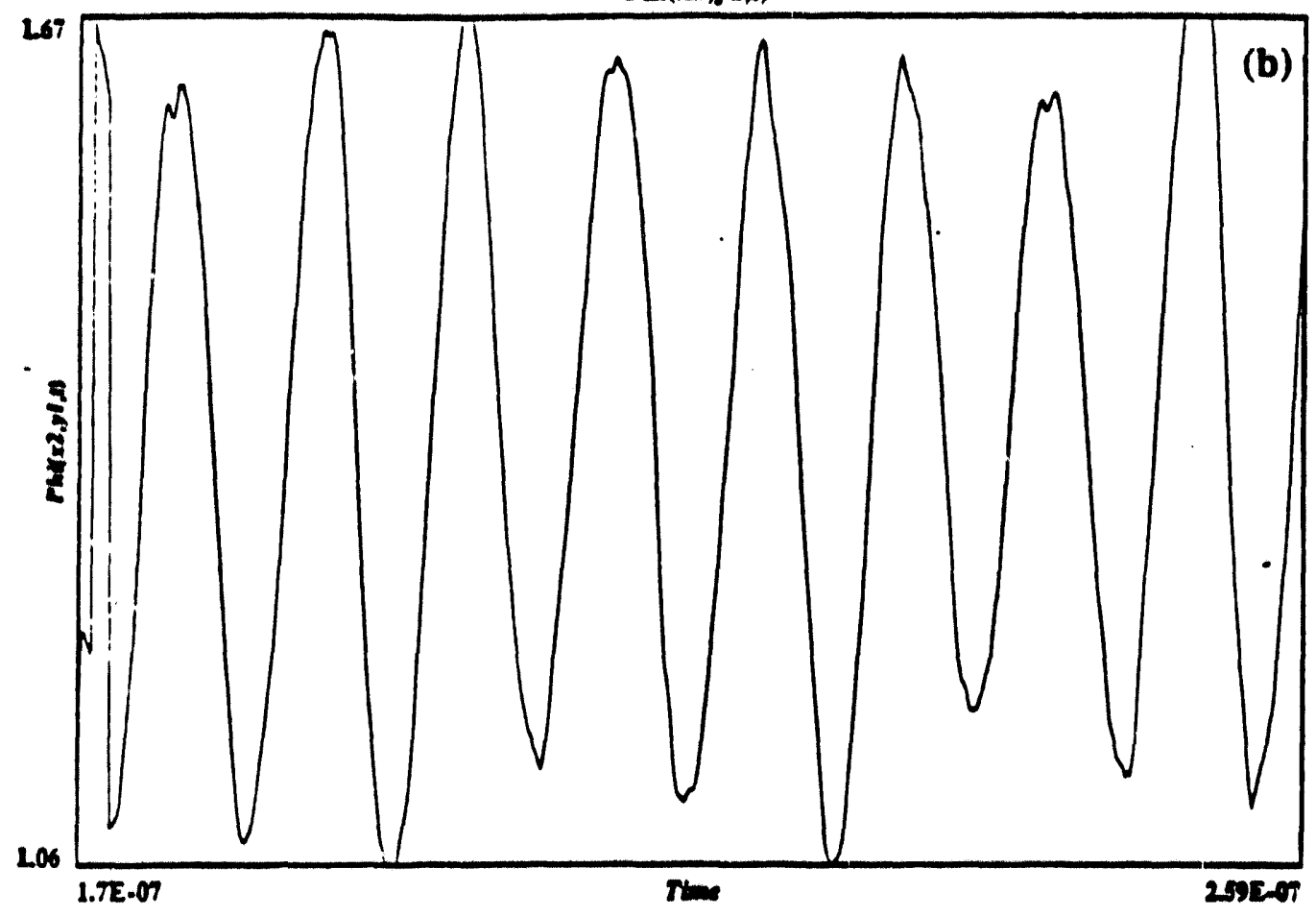

$\operatorname{Phi}(\times 2, y 2: 1)$

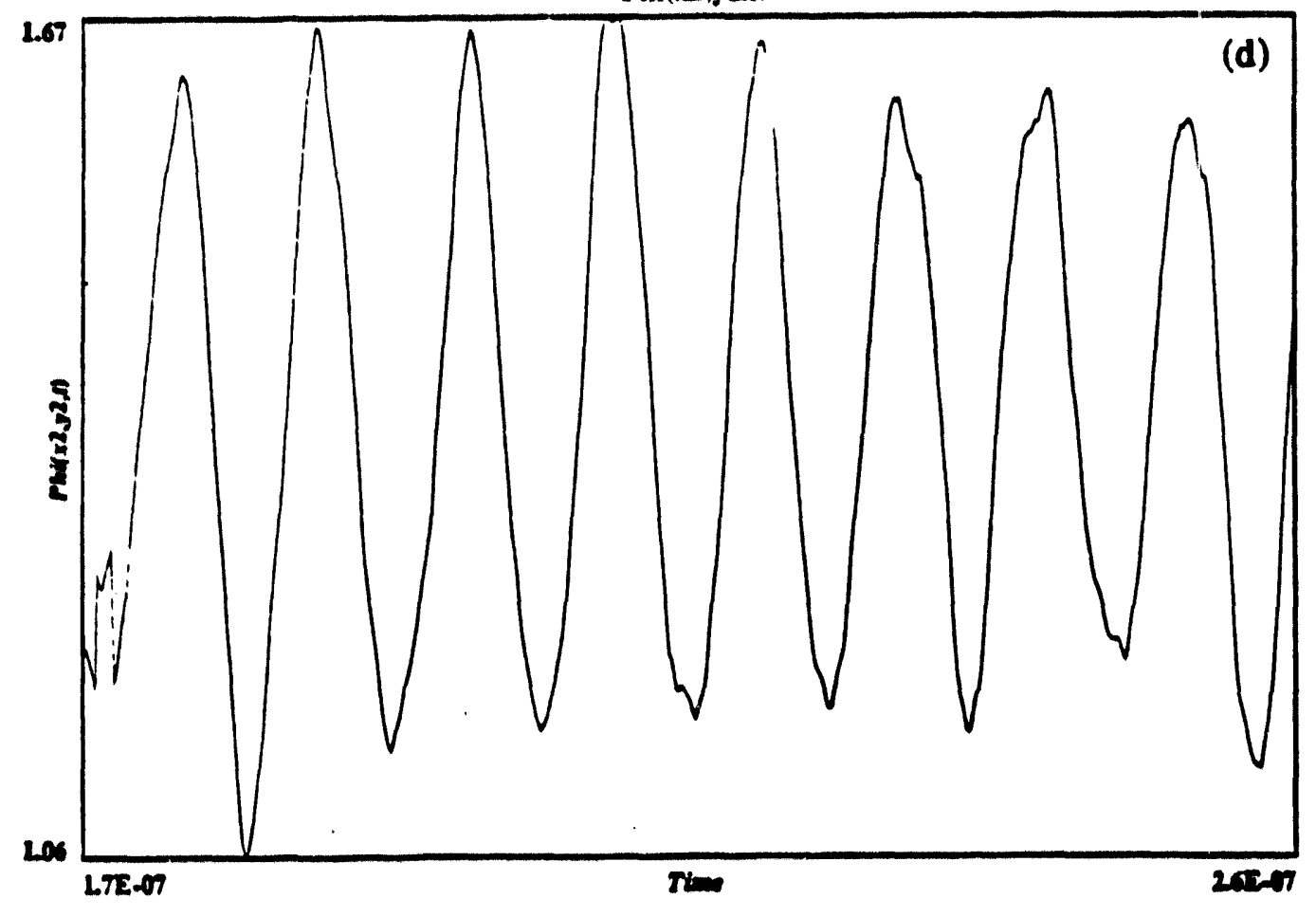

Figure 6: The time records of a pulsing on and of a DC potential a two bontions. Fig $6 \mathrm{~b}$. Sourice $x_{2}$

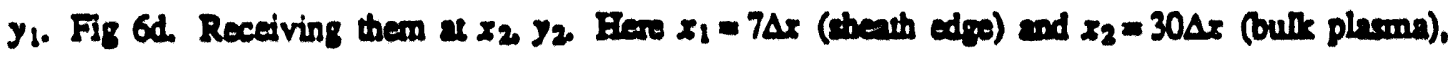
$y_{1}-y_{2} \equiv L_{3} / 2$. Bulk plasma cocillations dominare in b and $d$ 
no conclusive results.

Third, we pulsed on five cycles of RF at roughly twice $\omega_{\text {pes }}$ and looked for the response downstream. The result are shown in Fig. 7. For $k_{y}=0$, we saw very clear results; in the bulk region, the potential had a very strong response at about $\omega_{\text {pei }}$ in the sheath region, we saw a very strong response at about $\omega_{p e} / 2$, taken to be either the series resonance or that at the local $\omega_{p e}$. For the smalleat non-zero wavenumber, $k_{y} L_{y}=2 \pi$, we could not make out any clear-cut response. Fig. 8 shows the same spectra as in Fig. 7 , at a later time interval, without the five cycles of $2 \omega_{\text {pe }}$ excitation. The conclusion from these two figures is that the Bohm-Gross waves appear in the bulk, but not in the sheath region, and the sheath waves appear in the sheath, but not in the bulk.

Lastly, back to what was done in 1991, we looked at the spectra of the potential with no drive. (There is sufficient noise in the system to drive all natural responses). We have observed the plasma and the series resonance responses in $1 \mathrm{~d}$ simulations; the $k_{y}=0$ results in $2 \mathrm{~d}$ must give the same, and did. For the smallest wavenumber, $k_{y} L_{y}=2 \pi$, we could not make out any clear-cut response, a large disappointment indeed.

\section{Conclusion}

We have found electrostatic surface waves between the sheath and plasma interface. It can be seen that PDP2 simulations and theory compare fairly well for both sheath waves and Bohm-Gross waves in the bulk for our simple model for $k_{y}=0$. But there is no conclusive comparisons for $k_{y} \neq 0$. The simulations must have better wave-detecting diagnostics, such as spatial correlations. The theory must allow for spatial variation of the zero-order density, as done in Id by Parker, Nickel and Gould. At least, we have given it a good try, and left something for those who are to follow. The next step is to study sheath waves for a magnetized sheath.

\section{Acknowledgments}

We thank Dr. R. B. Coben (LLNL) for enlightening discussions and for useful suggestions from Prof. R. W. Gould (Cal. Tech.). This work was performed at the University of Californis at Berkeley under U. S. Office of Naval Research contract N00014-85-K0809 and U. S. Department of Energy contract DE-FG03-90ER54079. 

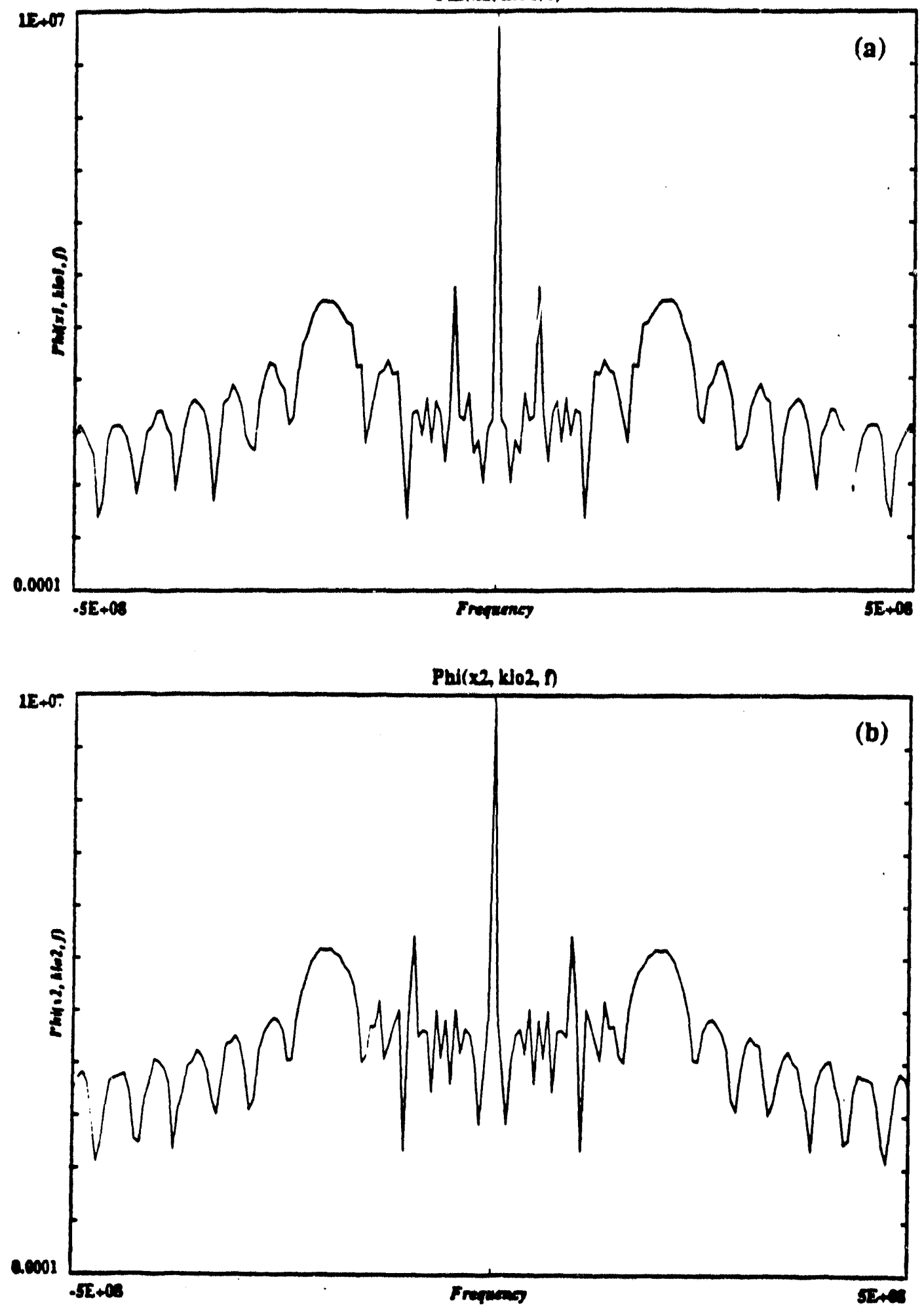

Figure 7: The power spectrum for a $2 d$ particle simularion of sheath waves ater pulsing on five cycles of RF a roughly twice $w_{m}$. Fig 72 . The power spectrum of $k_{y}=0$ mode $a$ streach edfe $x=7 \Delta x$; the shrp spikes are taken to be the series resonences. Fig 70 . The power spectrum of $k_{y}=$ mode in the

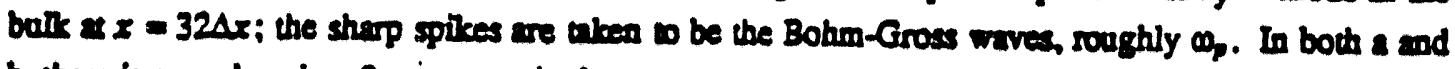
b. there is a road peak a $2 \omega_{\text {min }}$ a excired 
Puil(xl, khil, n

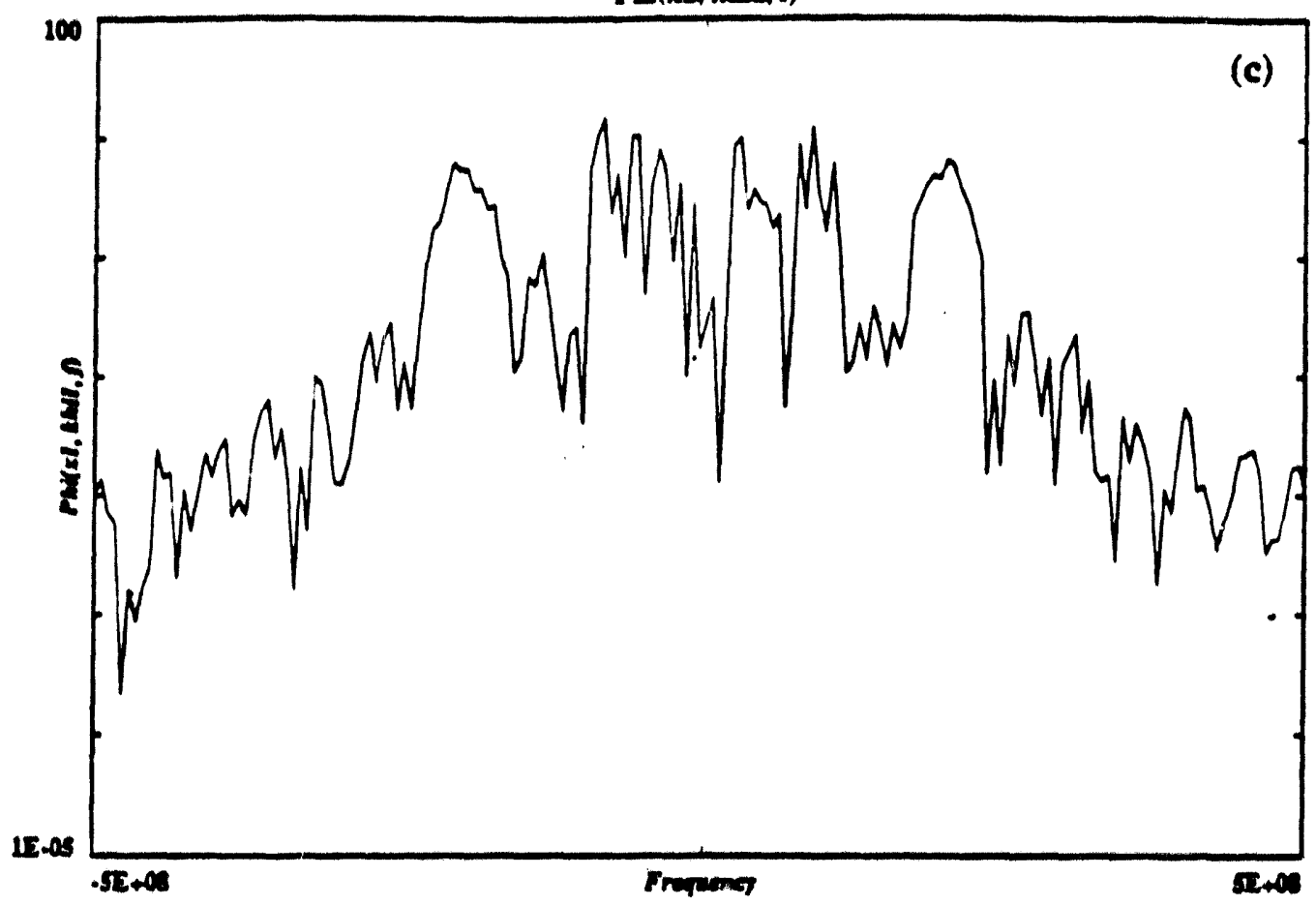

Pul(x2, knith, n

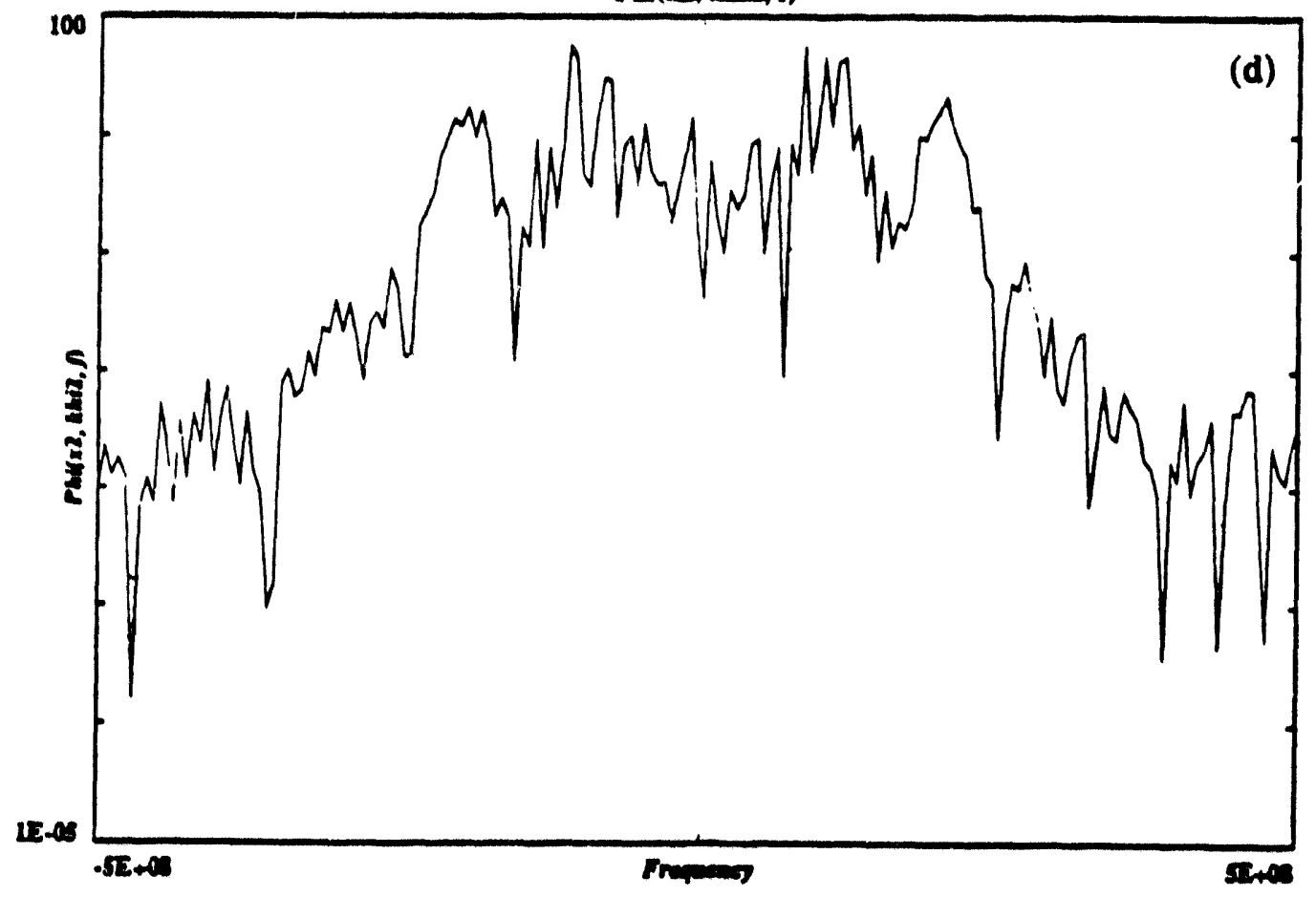

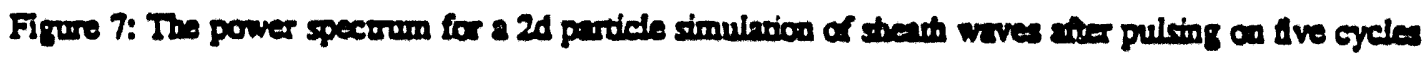

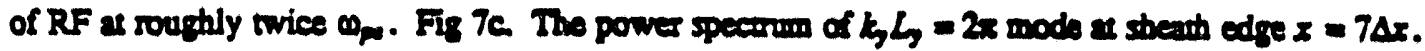

Fig 7d. The power speceruen of $k, h_{2}=2 x$ mode in the bulk $x x=32 A x$. 

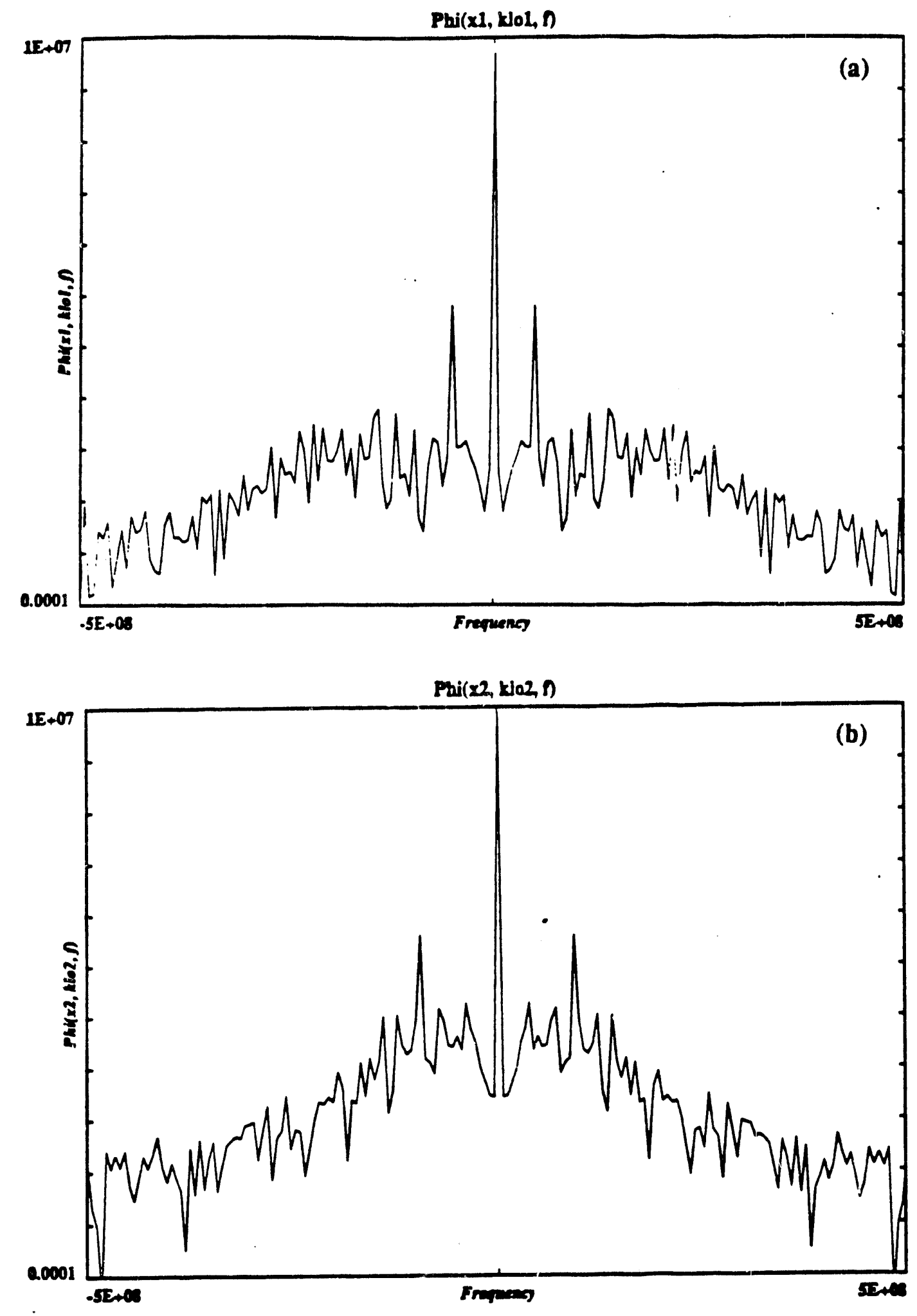

Figure 8: The same order as Figure 7, generated at later time. The FFT interval now did not include the 3 cycles at 2 ipe. Bence, these spectrom may be taken as the antural response of plasmen, simply due to excitation by noise Note that (a) has a strong response at series resonance and (b) at planms resonance. 


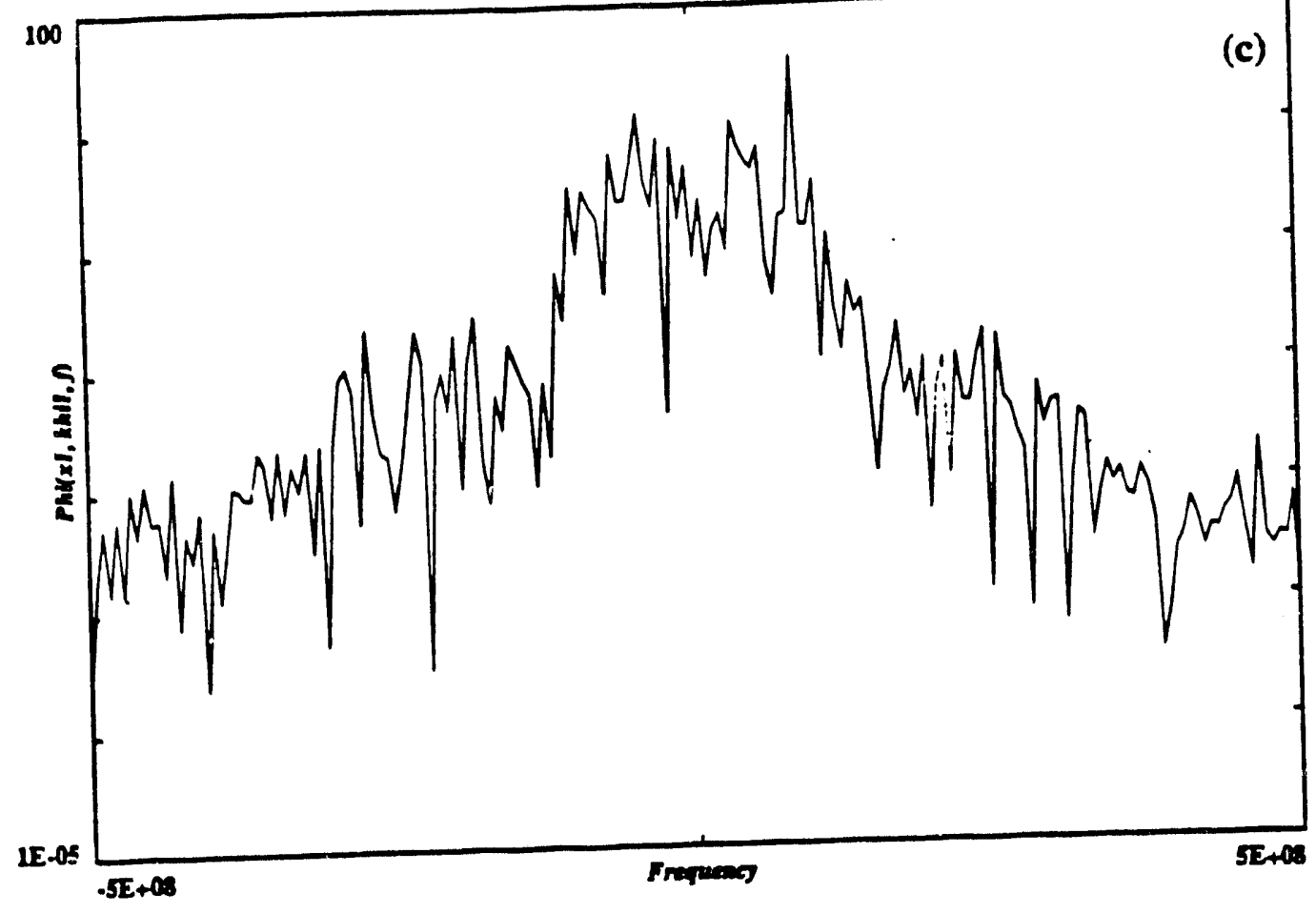

$\operatorname{Phi}\left(x_{2}\right.$, khi2, n

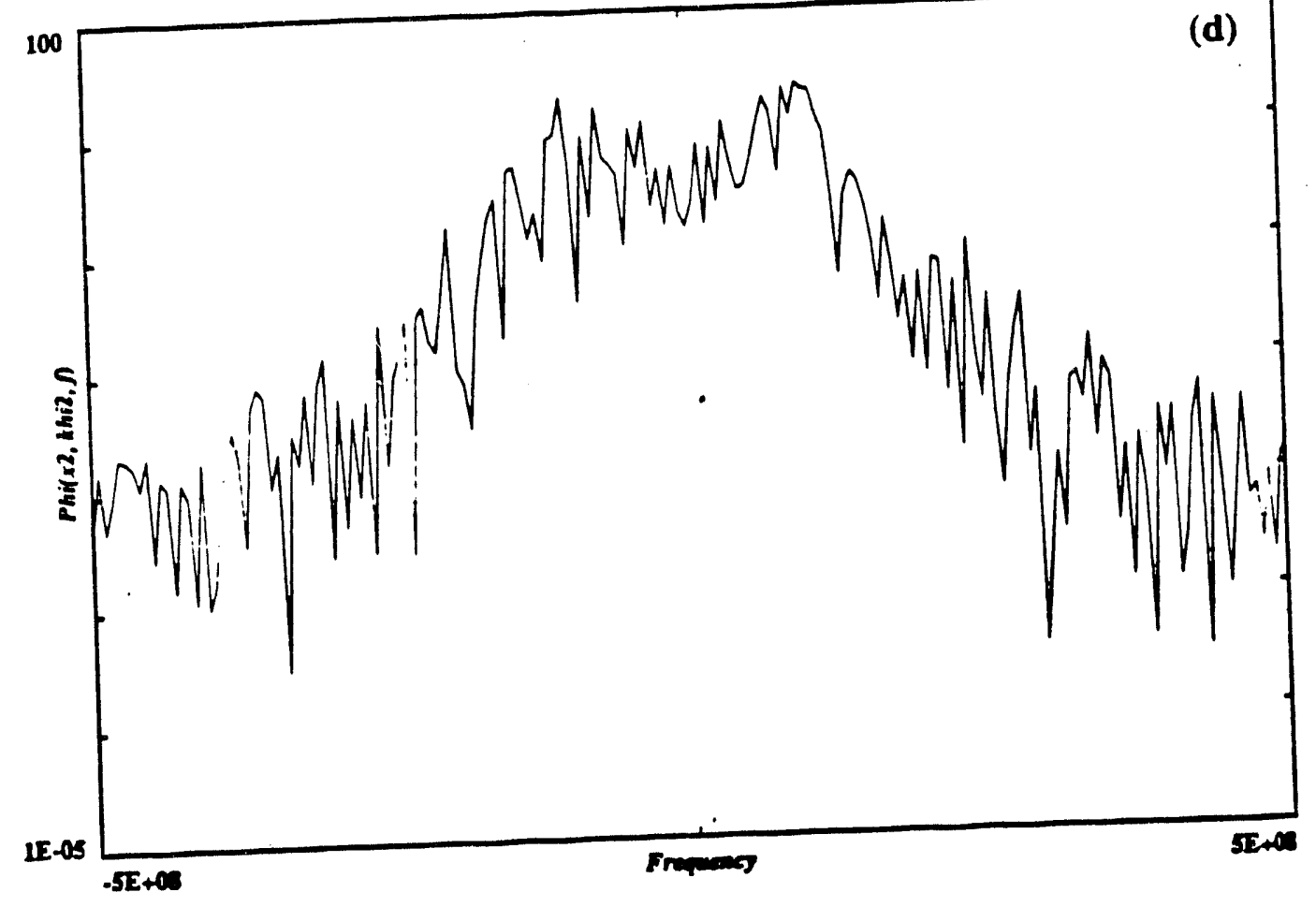

Figure 8: The same order as Figure 7, generated at later time. The FIT interval now did not include the 5 cyeles at $2 \omega_{\text {per. }}$. Bence, these spectrum may be taken as the satural response of plasma, simply due to excitation by noise. Note that (a) bes a strong response at ceries resonance and (b) at plasma resonance. 


\section{References}

[1] V. Vahedi, private communication.

[2] R. S. Harp and F. W. Crawford, Journal of Applied Physics 35, 3436 (1964); H. M. Mayer, Proceeding of VIth International Conference on Jonization Phenomena in Gases (Paris, July 1963) (S. E. R. M. A. Publishing Company, Paris, 1964), Vol. 4, p.129; R. W. Gould, Phys. Letters 11,236 (1964).

[3] A. W. Trivelpiece, Slow Wave Propagation in Plasma Wave Guides, San Francisco Press, San Francisco, 1967.

[4] V. K. Decyk, J. M. Dawson, J. Comp. Phys. 30, 407 (1979).

[5] C. C. Cheng and E. G. Harris Phys. of Fluids 12, 1262 (1968).

[6] Y. Takase, et al., Phys. Rev. Lett 59, 1201 (1987).

[7] J. R. Myra, D. A. D'Ippolito, D. W. Forslund, and J. U. Brackbill, Phys. Rev. Lett 66, 1173 (1991);

[8] A. G. Sitenko, Fluctuations and Non-linear wave Interactions in Plasmas, Pergramon Press, Oxford, 1982.

[9] H. L. Berk, D. D. Ryutov and Yu. Tsidulko, Phys. of Fluids 3, 1364 (1991).

[10] C. K. Birdsall and A. B. Langdon, Plasma Physics via Computer Simulation, (McGrawHill, New York, 1985 and Adam Hilger, 1991).

[1.1] K. Theilhaber and C. K. Birdsall, Phys. Rev. Lett 62, 772 (1989); K. Theilhaber and C. K. Birdsall, Phys. of Fluids B1, 2241 (1989); K. Theilhaber and C. K. Birdsall, Phys. of Fluids B1, 2260 (1989).

[12] V. I. Okulov, in Surface waves in Plasmas and Solids, edited by S. Vukovic, (World Scientific, 1986), p.618. 

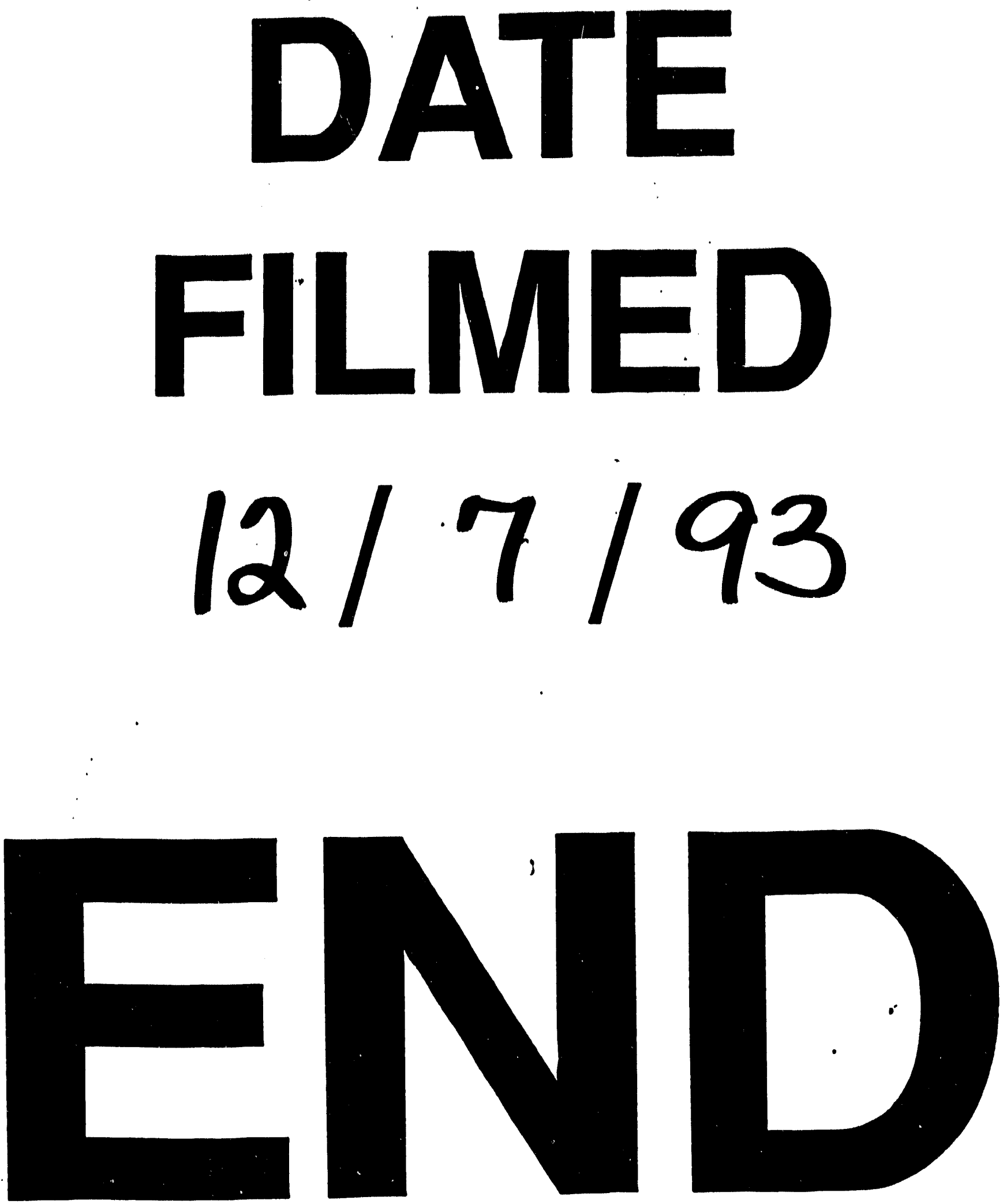
